\title{
The overexpression of TDP-43 in astrocytes causes neurodegeneration via a PTP1B- mediated inflammatory response
}

Shinrye Lee ${ }^{1 \dagger}$, Seyeon Kim ${ }^{1,2+}$, Ha-Young Kang ${ }^{3}$, Hye Ryeong Lim ${ }^{4}$, Younghwi Kwon ${ }^{1,2}$, Myungjin Jo', Yu-Mi Jeon ${ }^{1}$, Sang Ryong Kim, ${ }^{5,6}$ Kiyoung Kim, Chang Man Ha ${ }^{4}$, Seongsoo Lee ${ }^{3^{*}}$ and Hyung-Jun Kim ${ }^{{ }^{*}}$

\begin{abstract}
Background: Cytoplasmic inclusions of transactive response DNA binding protein of $43 \mathrm{kDa}$ (TDP-43) in neurons and astrocytes are a feature of some neurodegenerative diseases, such as frontotemporal lobar degeneration with TDP-43 (FTLD-TDP) and amyotrophic lateral sclerosis (ALS). However, the role of TDP-43 in astrocyte pathology remains largely unknown.

Methods: To investigate whether TDP-43 overexpression in primary astrocytes could induce inflammation, we transfected primary astrocytes with plasmids encoding Gfp or TDP-43-Gfp. The inflammatory response and upregulation of PTP1B in transfected cells were examined using quantitative RT-PCR and immunoblot analysis. Neurotoxicity was analysed in a transwell coculture system of primary cortical neurons with astrocytes and cultured neurons treated with astrocyte-conditioned medium (ACM). We also examined the lifespan, performed climbing assays and analysed immunohistochemical data in pan-glial TDP-43-expressing flies in the presence or absence of a Ptp61f RNAi transgene.

Results: PTP1B inhibition suppressed TDP-43-induced secretion of inflammatory cytokines (interleukin 1 beta (IL-1 $\beta$ ), interleukin 6 (IL-6) and tumour necrosis factor alpha (TNF-a)) in primary astrocytes. Using a neuron-astrocyte coculture system and astrocyte-conditioned media treatment, we demonstrated that PTP1B inhibition attenuated neuronal death and mitochondrial dysfunction caused by overexpression of TDP-43 in astrocytes. In addition, neuromuscular junction (NMJ) defects, a shortened lifespan, inflammation and climbing defects caused by pan-glial overexpression of TDP-43 were significantly rescued by downregulation of ptp61f (the Drosophila homologue of PTP1B) in flies.
\end{abstract}

Conclusions: These results indicate that PTP1B inhibition mitigates the neuronal toxicity caused by TDP-43-induced inflammation in mammalian astrocytes and Drosophila glial cells.

Keywords: Neurodegenerative disease, Neuroinflammation, Astrocytes, Tar DNA-binding protein 43, Protein tyrosine phosphatase 1B

\footnotetext{
* Correspondence: soolee@kbsi.re.kr; kijang1@kbri.re.kr

†Shinrye Lee and Seyeon Kim contributed equally to this work.

${ }^{3}$ Gwangju Center, Korea Basic Science Institute (KBSI), Gwangju 61886, South

Korea

'Dementia Research Group, Korea Brain Research Institute (KBRI), Daegu

41062, South Korea

Full list of author information is available at the end of the article
}

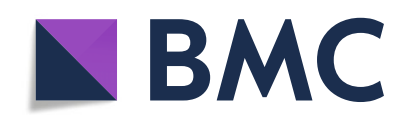

(- The Author(s). 2020 Open Access This article is licensed under a Creative Commons Attribution 4.0 International License, which permits use, sharing, adaptation, distribution and reproduction in any medium or format, as long as you give appropriate credit to the original author(s) and the source, provide a link to the Creative Commons licence, and indicate if changes were made. The images or other third party material in this article are included in the article's Creative Commons licence, unless indicated otherwise in a credit line to the material. If material is not included in the article's Creative Commons licence and your intended use is not permitted by statutory regulation or exceeds the permitted use, you will need to obtain permission directly from the copyright holder. To view a copy of this licence, visit http://creativecommons.org/licenses/by/4.0/ The Creative Commons Public Domain Dedication waiver (http://creativecommons.org/publicdomain/zero/1.0/) applies to the data made available in this article, unless otherwise stated in a credit line to the data. 


\section{Background}

Transactive response DNA binding protein of $43 \mathrm{kDa}$ (TDP-43) is a major component of cytoplasmic aggregates in neurons and glia in most patients with amyotrophic lateral sclerosis (ALS) and in a subgroup of patients with frontotemporal lobar degeneration with TDP-43 (FTLDTDP) [1-3]. TDP-43 is a type of heterogeneous nuclear ribonucleoprotein (hnRNP) that is ubiquitously expressed and concentrated in the nucleus [4]. More than 50 missense mutations in TARDBP have been identified in sporadic and familial cases of ALS [5]. Many transgenic animal models expressing wild-type (WT) or mutant TDP-43 have been generated, most of which mimic key clinical features found in ALS patients, such as impaired motor function, neurodegeneration and accumulation of cytoplasmic TDP-43 aggregates [6-10].

Cytoplasmic aggregation of TDP-43 is one of the major features in TDP-43 proteinopathy [1, 11, 12], and these aggregates are associated with many neurodegenerative diseases, including FTLD, ALS and Alzheimer's disease (AD) $[3,13,14]$. This cytoplasmic accumulation of TDP-43 eventually leads to neuronal toxicity. Several lines of evidence indicate that TDP-43 is ubiquitously expressed in many tissues and cell types, including glial cells of the central nervous system. The inflammatory activation of astrocytes and/or microglia is prevalent in most animal models of TDP-43 proteinopathy, such as disease-associated transgenic mice with mutations in TDP-43 and SOD1 [15]. Moreover, several studies have shown that the expression of mutant SOD1 in astrocytes and microglia significantly exacerbates neurodegeneration [16-20]. In particular, selective expression of TDP43 in rat astrocytes also leads to non-cell autonomous neuronal toxicity [21]. These data suggest that nonneuronal cells, such as microglia and astrocytes, contribute to neuronal toxicity in TDP-43 proteinopathy.

Protein tyrosine phosphatase 1B (PTP1B) regulates many important signalling pathways that are relevant to ALS, such as inflammation and ER stress. Previous studies suggest that inhibition of PTP1B is associated with early signalling in macrophages in response to inflammation [22]. Moreover, IL-4-induced anti-inflammatory features are negatively regulated by PTP1B [23]. PTP1B is also associated with microglia-mediated neuroinflammation. Recent studies suggest that high-fat diet-induced activation of hypothalamic microglia is significantly attenuated by PTP1B deficiency [24]. LPS-induced neuroinflammation in microglia is also mitigated by PTP1B inhibition [25]. In addition, a recent study indicated that ER stress-induced neuronal toxicity is dramatically reduced by PTP1B inhibition in Drosophila and mammalian neurons [26]. However, it has never been determined whether PTP1B is implicated in the proinflammatory activation of astrocytes.
In the present study, we found that PTP1B expression in astrocytes was upregulated by TDP-43 overexpression and that PTP1B inhibition attenuated the TDP-43induced proinflammatory response of astrocytes. By utilizing a pan-glial TDP-43 proteinopathy Drosophila model and mouse primary cell culture model, we showed that PTP1B is a critical mediator of the neuronal toxicity caused by TDP-43-induced neuroinflammation. Therefore, targeting PTP1B may represent a promising therapeutic intervention for neurodegenerative diseases with TDP-43 proteinopathy.

\section{Methods \\ Reagents}

The following reagents were purchased as indicated: dimethyl sulfoxide (DMSO) and all-trans retinoic acid (RA) [Sigma]; PTP1B inhibitor [Calbiochem/MerckMillipore]; and recombinant mouse IL-1 $\beta$ protein, recombinant mouse IL- 6 protein, and recombinant mouse TNF- $\alpha$ protein $[R \& D$ Systems].

\section{Antibodies}

The following antibodies were used for immunoblotting: mouse anti-TurboGFP (TA150041) [Origene]; rabbit anti-TDP-43 (10782-2-AP) [Proteintech]; mouse antiLamin A/C (05-714) [EMD Millipore]; rabbit anti-beta Actin (ab16039) [Abcam]; rabbit anti-NF- kB p65 (Ser536) (3033), rabbit anti-NF-kB (8242) and HRPconjugated anti- $\alpha$-tubulin (9099) [Cell Signaling Technology]; and rabbit anti-PTP1B (sc-14021), HRPconjugated anti-rabbit IgG (sc-2004) and HRPconjugated anti-mouse IgG (sc-2005) [Santa Cruz]. The following antibodies were used for immunocytochemistry (ICC): rabbit anti-MAP2 (1:500; AB5622) and TRIT C-conjugated phalloidin (1:500; 90228) [Millipore]. The following antibody was used for immunohistochemistry (IHC): FITC-conjugated anti-HRP (1:150; 123-095-021) [Jackson ImmunoResearch Laboratories]. The following antibodies were used for neutralizing target proteins: rabbit anti-IL-1 $\beta$ (ab9722) [Abcam]; rat anti-TNF- $\alpha$ (147321-81) [Invitrogen]; rabbit anti-IgG (2729) and mouse anti-IgG (5415) [Cell Signaling Technology]; and mouse anti-IL-6 (sc-57315) and rat anti-IgG (sc-2026) [Santa Cruz].

\section{Primary cell cultures}

Primary cultures of dissociated cerebral cortical neurons were prepared from C57/BL6 16-day-old embryonic mice as described previously $[27,28]$. Briefly, mouse embryos were decapitated, and the brains were rapidly removed and placed in a culture dish containing HBSS (Gibco). Cortices were isolated, transferred to a conical tube and washed twice in HBSS (Gibco). Cortical tissues were enzymatically digested with prewarmed papain $(20$ 
units/ml) (Worthington Biochemical Corporation) and DNase I $(0.005 \%)$ for $30 \mathrm{~min}$ at $37{ }^{\circ} \mathrm{C}$. The tissues were mechanically dissociated (triturated) with $1000 \mu \mathrm{l}$ and $200 \mu$ pipette tips to obtain complete tissue homogenization. Cortical cells were centrifuged at $130 \times g$ for $10 \mathrm{~min}$ at room temperature, and the dissociated cells that were obtained were seeded onto plates coated with poly-D-lysine (Sigma-Aldrich) in neurobasal media containing $2 \mathrm{mM}$ glutamine (Gibco), N2 supplement (Gibco), B27 supplement (Gibco) and $50 \mu \mathrm{g} / \mathrm{ml}$ penicillin-streptomycin ( $\mathrm{P} / \mathrm{S}, \mathrm{Gibco})$. Neuronal purity was determined by calculating the ratio of MAP2positive cells to total viable cells (Figure S1a; upper). The culture media were changed initially after 5 days and every 3 days thereafter, and cells were used after being cultured for 14-21 days.

Primary astrocyte cultures were prepared from 1- to 2day-old C57/BL6 mice as described previously [29]. Briefly, whole brains were homogenized and passed through a $70-\mu \mathrm{m}$ strainer. Cells were seeded in T75 culture flasks. Cells were grown at $37{ }^{\circ} \mathrm{C}$ in a humidified atmosphere containing $5 \% \mathrm{CO}_{2}$. Culture medium was changed initially after 5 days and every 2 days thereafter, and cells were used after being cultured for 14-21 days. Secondary pure astrocyte cultures were obtained by shaking mixed glial cultures at $250 \mathrm{rpm}$ for $4 \mathrm{~h}$; then, the culture medium was discarded. Astrocytes were dissociated using trypsin-EDTA (Life Technologies) and then were centrifuged at $800 \times g$ for $30 \mathrm{~min}$. The astrocytes obtained were seeded onto plates in DMEM (Life Technologies) supplemented with 10\% heat-inactivated foetal bovine serum (FBS; Gibco) and $50 \mu \mathrm{g} / \mathrm{ml} \mathrm{P} / \mathrm{S}$. The purity of the cells in culture was determined by immunocytochemistry, which found that the cultures contained over 93\% GFAP-positive cells (Figure S1a; lower). Animals used in the current research were acquired and cared for in accordance with the guidelines published in the National Institutes of Health Guide for the Care and Use of Laboratory Animals.

\section{Transfection}

Primary astrocytes in 6-well plates $\left(40 \times 10^{4}\right.$ cells/well $)$ were transfected with $4 \mu \mathrm{g}$ of Gfp ( $p C M V 6-A C$-Gfp, Origene Technologies, PS100010) or human TDP-43 (pCMV6-AC-TDP-43-Gfp, Origene Technologies, RG210639) vectors using Lipofectamine 3000 reagent (Invitrogen). At 2 days posttransfection, cells were subjected to FACS of Gfp-transfected live cells. The Gfptransfected sorted cells $\left(8 \times 10^{4}\right.$ cells/well $)$ were treated with a PTP1B inhibitor (PTP1Bi, $5 \mu \mathrm{M}$ ) or DMSO for 24 h. A total experimental duration of 3 days posttransfection was chosen to reduce the transfection-associated background toxicity.

\section{siRNA transfection}

Primary astrocytes in 6-well plates $\left(40 \times 10^{4}\right.$ cells/well $)$ were cotransfected with a $p C M V 6-A C-T D P-43-G f p$ vector and control siRNA (Dharmacon; D-001810-10) or mouse Ptb1b siRNA (Dharmacon; L-040818-00) using Lipofectamine 3000 reagent (Invitrogen) or Lipofectamine RNAiMAX reagent (Invitrogen), and then the cells were incubated for 3 days. The downregulation of target protein expression in the transfected cells was confirmed by immunoblot analysis. At $72 \mathrm{~h}$ posttransfection, cells were subjected to FACS of Gfp-transfected live cells, and the sorted cells were then fixed or harvested for further analyses.

\section{Cytotoxicity tests}

Primary astrocytes $\left(5 \times 10^{4}\right.$ cells/well $)$ were grown in 96well plates and treated with a PTP1B inhibitor (PTP1Bi, $5 \mu \mathrm{M})$ for $24 \mathrm{~h}$. Cells cultured in an equal volume of DMSO were used as a control. To measure cytotoxicity, Cell Counting Kit-8 (CCK-8; Enzo Life Science) was used in accordance with the manufacturer's instructions. Briefly, $10 \mu \mathrm{l}$ of CCK- 8 reagent was added to each well, and the plate was incubated at $37^{\circ} \mathrm{C}$ for $2 \mathrm{~h}$. Absorbance was measured at $450 \mathrm{~nm}$ using a microplate reader (Tecan). Cell viability was expressed as a percentage of control (DMSO-treated) cell viability. All experiments were performed in triplicate.

\section{Quantitative RT-PCR}

RNA was extracted from cells and fly heads using TRIzol reagent (Life Technologies). RNA cleanup was performed using an RNeasy Mini Kit (QIAGEN) according to the manufacturer's instructions. cDNA synthesis was performed at $37{ }^{\circ} \mathrm{C}$ for $120 \mathrm{~min}$ with $100 \mathrm{ng}$ of RNA using a High Capacity cDNA Reverse Transcription Kit (Applied Biosystems). Quantitative RT-PCR was performed using a One-Step SYBR ${ }^{\circ}$ PrimeScript ${ }^{\mathrm{tm}}$ RT-PCR Kit (Perfect Real Time; Takara Bio Inc.) according to the manufacturer's instructions, which was followed by detection using an Applied Biosystems 7500 Real-Time PCR system (Applied Biosystems). Gapdh and 18S rRNA were used as internal controls. The $2^{-\Delta \Delta \mathrm{Ct}}$ method was used to calculate relative differences in gene expression that were determined by real-time PCR experiments [30].

\section{Immunoblot analysis}

For total protein extraction, either cells or 20 adult fly heads were homogenized in RIPA buffer (Cell Signaling Technology) or 1× LDS sample buffer (Invitrogen) containing a protease and phosphatase inhibitor cocktail (Roche). The protein concentration of the cell lysates was determined using a BCA protein assay kit (Thermo Fisher Scientific). Next, the protein extracts were mixed 
with $4 \times$ NuPAGE LDS sample buffer (Invitrogen) and 10× NuPAGE Sample Reducing Agent buffer (Invitrogen), and then they were boiled at $95{ }^{\circ} \mathrm{C}$ for $5 \mathrm{~min}$. An equal amount of protein from each sample was separated on NuPAGE 4-12\% Bis-Tris gels (Novex) or NuPAGE 3-8\% Tris-acetate gels (Novex) and then was transferred to a polyvinylidene difluoride (PVDF; Novex) membranes using a transfer apparatus according to the manufacturer's protocol (Novex). The membranes were blocked with $5 \%$ skim milk and were sequentially incubated with primary antibodies and HRP-conjugated secondary antibodies (anti-rabbit IgG and anti-mouse IgG), followed by detection with an ECL Prime kit (Amersham Biosciences). Samples from three independent experiments were used in this analysis. The relative expression level was determined using Fusion-FX software (Vilber Lourmat).

\section{Nuclear and cytoplasmic fraction extraction}

TDP-43-transfected astrocytes $\left(15 \times 10^{4}\right.$ cells/well $)$ were fractionated using NE-PER nuclear and cytosolic extraction reagents (Thermo Fisher Scientific). Nuclear and cytoplasmic fractions in ice-cold CER I and CER II buffer were obtained by centrifugation at $16,000 \times \mathrm{g}$ for 5 min at $4{ }^{\circ} \mathrm{C}$. Supernatants containing the cytoplasmic extract were harvested, and the pellets were solubilized in ice-cold NER buffer. After vortexing, the extracts were centrifuged at $16,000 \times \mathrm{g}$ for $10 \mathrm{~min}$ at $4{ }^{\circ} \mathrm{C}$. Supernatants containing the nuclear extract were harvested. The extracts were mixed with $4 \times$ NuPAGE LDS sample buffer and 10× NuPAGE Sample Reducing Agent buffer and then were boiled at $95^{\circ} \mathrm{C}$ for $5 \mathrm{~min}$.

\section{Elisa}

To determine IL-1 $\beta$, IL- 6 and TNF- $\alpha$ protein levels, supernatants of TDP-43-transfected astrocytes $\left(15 \times 10^{4}\right.$ cells/well) were analysed using mouse ELISA Development Kits for each cytokine or chemokine (R\&D Systems). Briefly, 96-well ELISA plates were coated with the capture antibodies. After blocking those antibodies, samples or recombinant cytokine or chemokine standards were added. For detection, biotinylated detection antibodies were added, which was followed by incubation with streptavidin-HRP and substrate (R\&D Systems) according to the manufacturer's instructions. The absorbance was measured at $450 \mathrm{~nm}$ and $540 \mathrm{~nm}$ using a microplate reader (Tecan).

\section{Astrocyte-conditioned media}

To produce PTP1B inhibitor-treated astrocyteconditioned medium (ACM), Gfp/TDP-43-transfected live primary astrocytes $\left(15 \times 10^{4}\right.$ cells/well $)$ that were obtained via FACS were allowed to acclimate for $24 \mathrm{~h}$ in DMEM. Gfp- or TDP-43-transfected primary astrocytes were treated with a PTP1B inhibitor (PTP1Bi, $5 \mu \mathrm{M})$ or DMSO for $24 \mathrm{~h}$. Primary astrocytes were then washed twice with PBS and cultured in fresh DMEM for an additional $24 \mathrm{~h}$. The ACM was then collected, centrifuged at $200 \times g$ for 10 min to remove cell debris and stored at $80{ }^{\circ} \mathrm{C}$ until further analysis.

To generate Ptp1b siRNA-transfected ACM, live primary astrocytes $\left(15 \times 10^{4}\right.$ cells/well $)$ that were cotransfected with a Gfp/TDP-43 expression construct and a Ptp1b siRNA or control siRNA were selected via FACS were allowed to acclimate for $24 \mathrm{~h}$ in DMEM. Primary astrocytes were then washed twice with PBS and cultured in fresh DMEM for an additional $24 \mathrm{~h}$. The ACM was then collected, centrifuged at $200 \times g$ for $10 \mathrm{~min}$ to remove cell debris and stored at $-80^{\circ} \mathrm{C}$ until further analysis. To obtain the control ACM, cells were cultured in DMEM supplemented with $10 \% \mathrm{FBS}$ and $50 \mu \mathrm{g} / \mathrm{ml} \mathrm{P} / \mathrm{S}$.

To generate IL-1 $\beta$, IL- 6 or TNF- $\alpha$ antibodyneutralized ACM, Gfp/TDP-43-transfected live primary astrocytes $\left(15 \times 10^{4}\right.$ cells/well $)$ obtained via FACS were allowed to acclimate for $24 \mathrm{~h}$ in DMEM. Gfp- or TDP43-transfected primary astrocytes were treated with an IL-1 $\beta$ antibody ( $50 \mathrm{ng} / \mathrm{ml})$, an IL- 6 antibody $(50 \mathrm{ng} / \mathrm{ml})$, a TNF- $\alpha$ antibody $(100 \mathrm{ng} / \mathrm{ml})$ and a control IgG (100 $\mathrm{ng} / \mathrm{ml}$ ) for $1 \mathrm{~h}$. Primary astrocytes were then washed twice with PBS and cultured in fresh DMEM for an additional $24 \mathrm{~h}$. The ACM was then collected, centrifuged at $200 \times g$ for $10 \mathrm{~min}$ to remove cell debris and stored at $80^{\circ} \mathrm{C}$ until further analysis.

\section{ACM-treated neuron culture}

For ACM-treated neuron culture, primary cortical neurons were stimulated with Gfp-transfected + DMSOtreated ACM, Gfp-transfected + PTP1B inhibitor-treated ACM, TDP-43-transfected + DMSO-treated ACM and TDP-43-transfected + PTP1B inhibitor-treated ACM (GFP ACM, GFP + PTP1B ACM, TDP-43 ACM and TDP-43 + PTP1Bi ACM, respectively) for $24 \mathrm{~h}$, and then the cells were subjected to a CCK- 8 assay. Primary cortical neurons were also treated with $G f p+$ control siRNA, Gfp + Ptp1b siRNA, TDP-43 + control siRNA and TDP-43 + Ptp $1 b$ siRNA cotransfected ACM (GFP ACM, GFP + Ptp1b siRNA ACM, TDP-43 ACM and TDP-43 + Ptp1b siRNA ACM, respectively) for 5 days, and then the cells were subjected to a CCK- 8 assay.

\section{Neuron-astrocyte coculture}

For neuron-astrocyte coculture, Gfp/TDP-43-transfected live primary astrocytes $\left(8 \times 10^{4}\right.$ cells/well $)$ that were selected via FACS were allowed to acclimate for $24 \mathrm{~h}$ in fresh DMEM. Gfp/TDP-43-transfected primary astrocytes were treated with a PTP1B inhibitor (PTP1Bi, 5 $\mu \mathrm{M})$ or DMSO for $24 \mathrm{~h}$. Primary astrocytes were then washed twice with PBS, detached, and seeded in the 
upper compartment of transwell inserts $(8 \mu \mathrm{m}$ pore membrane; Millipore) at a density of $4 \times 10^{4}$ cells/well. Primary cortical neurons were plated in the lower compartment of 24-transwell plates at a density of $4 \times 10^{4}$ cells/well. The primary cells were incubated at $37^{\circ} \mathrm{C}$ for either 36 or $48 \mathrm{~h}$ and then were subjected to a CCK-8 assay.

\section{CMFDA staining analysis}

Chloromethylfluorescein diacetate (CMFDA) is a longterm cell-tracing green dye that efficiently stains all cells [31, 32]. A CMFDA fluorescence-based assay was used to quantify the relative changes in neuronal cell viability. Primary cortical neurons were labelled with a $5 \mu \mathrm{M}$ concentration of CellTracker Green CMFDA Dye (Invitrogen) for $30 \mathrm{~min}$ according to the manufacturer's protocol. Then, the dye solution was aspirated, and the cells were incubated with dye-free medium for $45 \mathrm{~min}$. The samples were mounted and observed with a microscope. Photomicrographs from three randomly chosen fields were obtained, and the number of CMFDApositive cells was counted. The quantification of CMFDA-positive cells is represented as a percentage of the control.

\section{NSC-34 cell culture}

NSC-34 cells (cat. no. CLU140; Cedarlane) are wellcharacterized lower motor neuron-like cells generated by the fusion of embryonic mouse spinal cord cells and mouse neuroblastoma cells [33]. NSC-34 cells show morphological and physiological similarities to mature primary motor neurons. NSC-34 cells were maintained in DMEM supplemented with $10 \% \mathrm{FBS}, 50 \mu \mathrm{g} / \mathrm{ml} \mathrm{P} / \mathrm{S}$ and $2 \mathrm{mM}$ glutamine. The differentiation of NSC-34 cells was performed as described previously $[34,35]$. For differentiation, NSC34 cells were grown to confluence, and the growth medium (DMEM + 10\% FBS) was exchanged for differentiation medium (1:1 DMEM/Ham's F12 supplemented with $1 \%$ FBS, $1 \%$ MEM-NEAA, $50 \mu \mathrm{g} / \mathrm{ml} \mathrm{P/S}$ and $1 \mu \mathrm{M}$ all-trans retinoic acid (RA)) every 2 days.

\section{Immunocytochemistry analysis}

Cells were fixed in $4 \%$ or $8 \%$ paraformaldehyde in PBS (Gibco, 70011-044) for $30 \mathrm{~min}$ at room temperature. The cells were then washed three times with PBS and permeabilized in PBS-T (0.3\% Triton X-100) for $15 \mathrm{~min}$ at room temperature. After blocking with $10 \%$ normal goat serum in PBS-T for $1 \mathrm{~h}$, primary antibodies and $2 \%$ normal goat serum in PBS-T were incubated with the cells overnight at $4{ }^{\circ} \mathrm{C}$. The cells were then washed three times with PBS-T and incubated with an Alexaconjugated secondary antibody for $1 \mathrm{~h}$ at room temperature. Alexa 594-conjugated goat anti-rabbit IgG (1:500; Jackson ImmunoResearch Laboratories, 111-585-
144), Alexa 488-conjugated anti-rabbit IgG antibody (1: 500; Jackson ImmunoResearch Laboratories, 111-545144), Alexa 594-conjugated anti-mouse IgG antibody (1: 500; Jackson ImmunoResearch Laboratories, 111-585146) and Alexa 647-conjugated anti-goat IgG antibody (1:500; Jackson ImmunoResearch Laboratories, 705-605147) were used as secondary antibodies as indicated. Then, samples were mounted and observed with a fluorescence microscope (Nikon).

\section{Mitochondrial activity assay}

For assessment of neuronal mitochondrial dysfunction, primary cortical neurons that were grown in XF24-well culture plates (Seahorse Bioscience) were stimulated with GFP ACM, TDP-43 ACM or TDP-43/PTP1B inhibitor-treated ACM for 5 days. After the treatments, the cells were washed twice with XF Base Medium supplemented with $2 \mathrm{mM}$ L-glutamine, $10 \mathrm{mM}$ D-glucose and $1 \mathrm{mM}$ sodium pyruvate $(\mathrm{pH} 7.4)$ and incubated at $37{ }^{\circ} \mathrm{C}$ in a non- $\mathrm{CO}_{2}$ incubator for $1 \mathrm{~h}$. Mitochondrial dysfunction was evaluated using an XF Cell Mito Stress Test Kit (Seahorse Bioscience) according to the manufacturer's instructions, followed by measurement using an XF24 Extracellular Flux Analyzer (Seahorse Bioscience). The 24-well utility plate was hydrated, treated with $2 \mu \mathrm{M}$ oligomycin, $1 \mu \mathrm{M}$ carbonyl cyanide 4-(trifluoromethoxy) phenylhydrazone (FCCP), $0.5 \mu \mathrm{M}$ antimycin A+rotenone, and then calibrated by the analyser. The basal oxygen consumption rate (OCR), ATP production, maximum reserve and respiratory capacity were calculated as previously described [36], and averages were calculated from five wells per condition in each individual experiment. The OCR was normalized to the total protein concentration (OD). After the seahorse analysis, the plate was centrifuged at $280 \times g$ for $5 \mathrm{~min}$. The media were aspirated and washed twice with PBS. Cells were lysed in RIPA buffer. Protein concentrations of cell lysates were determined using a BCA assay kit.

\section{Quantification of dendritic spines}

Cells were fixed in 8\% paraformaldehyde in PBS (Gibco) for $30 \mathrm{~min}$ at room temperature. The cells were then washed three times with PBS and permeabilized in PBS$\mathrm{T}(0.3 \%$ Triton $\mathrm{X}-100)$ for $15 \mathrm{~min}$ at room temperature. After blocking with $10 \%$ normal goat serum in PBS-T for $1 \mathrm{~h}$, a MAP2 antibody with $2 \%$ normal goat serum in PBS-T was incubated with the cells overnight at $4{ }^{\circ} \mathrm{C}$. The cells were then washed three times with PBS-T and incubated with TRITC-conjugated phalloidin and an Alexa 594-conjugated goat anti-rabbit IgG (1:500) for 1 $\mathrm{h}$ at room temperature. Then, samples were mounted and observed with a confocal microscope (Nikon). Fifteen dendrites were randomly selected from four separate cortical neuron cultures in the GFP ACM, TDP-43 
ACM and TDP-43 + PTP1Bi ACM-treated cortical neuron groups. Images of dendritic spine were taken from 7 raw images of 4 independent primary cortical neuron cultures per ACM-treated group, and 1 or $2 \mathrm{im}$ ages were excluded from each group because the spine analysis was disturbed by crossing dendrite images. Each image was acquired to z-stack image series, which included 15 frames with $200 \mathrm{~nm}$ sections using a $\times 100$ objective (FOV 1024 BY 1024) with a Nikon A1Rsi confocal microscope. The $\mathrm{z}$-stack image that was selected contained a dendritic segment approximately $50 \mu \mathrm{m}$ in length that was distal to a dendritic branch point, and it was manually analysed for additional dendritic branch points. Dendritic spines were analysed for dendritic protrusions that could not be classified as stubby, mushroom, thin spine or filopodia. The maximum spine length and minimum spine end diameter were set at 5 $\mu \mathrm{m}$ and $0.2 \mu \mathrm{m}$, respectively. The dendritic spine morphological changes were analysed using Imaris software (Imaris, Bitplane, Inc.), and data were exported into Excel (Microsoft). Statistical analysis was then conducted using Prism 8 (GraphPad Prism Software, La Jolla, CA). All values are presented as the mean $\pm \mathrm{SEM}$, with $N$ indicating the number of replicates. The corresponding $p$ values are described in the figure legend of each figure.

\section{Fly strains}

Drosophila stocks were maintained on standard cornmeal agar media at $24{ }^{\circ} \mathrm{C}$ unless otherwise noted. The construct UAS-TDP-43 was described previously [11]. Repo-Gal4/+ and Repo-Gal4/UAS-TDP-43 have previously been described [37]. The upstream activation sequence-RNA interference (UAS-RNAi) line against Ptp61f or Egfp was obtained from the Bloomington stock centre (Bloomington, IN; http://flystocks.bioindiana.edu/ ). We crossed females from UAS-RNAi lines (Egfp

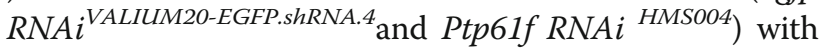
males harbouring the Repo-Gal4 (pan-glial) driver to knock down target genes in the entire glial cell population.

\section{Immunohistochemical analysis}

Wandering third instar larvae of adult flies were randomly selected, dissected in PBS and then fixed in $4 \%$ formaldehyde in PBS for approximately $15 \mathrm{~min}$. After blocking with $5 \%$ normal goat serum in PBS-T $(0.3 \%$ Triton X-100) for $1 \mathrm{~h}$, the antibodies with $5 \%$ normal goat serum in PBS-T were incubated with the fixed larvae for approximately $1.5 \mathrm{~h}$ at room temperature. Larval preparations were mounted with a SlowFade Antifade Kit (Invitrogen). NMJ images were visualized using a laser scanning confocal microscope system (TCS SP5 AOBS/Tandem microscope, Leica-Microscope Systems $\mathrm{GmbH}$, Germany) at Korea Basic Science Institute,
Gwangju Center. Leica Application Suite Advanced Fluorescence software was used to analyse images. We performed the analyses of NMJs essentially as described [38].

\section{Lifespan and adult climbing assays}

We performed lifespan and climbing assay using offspring from the crosses of Egfp RNAi ${ }^{\text {VALIU- }}$ M2O-EGFP.shRNA.4 or Ptp61f RNAi HMSO042 with Repo-Gal4 lines. Adult males ( 0 to 1 day old) were separated and transferred into experimental vials at a density of 20 (for lifespan) or 25 (for climbing assay) flies per vial $(n>$ 100). The number of dead flies was scored daily, and flies were transferred to fresh media or paper every other day. Adult locomotor function was assessed by a previously described method [39], and there were 125 flies per genotype per time point in all experiments. Experiments were repeated twice to ensure consistent results.

\section{Statistical analyses}

Data were analysed by Student's $t$ test (Vassar Stats, www.vassarstats.net) or were first analysed using oneway ANOVA followed by Bonferroni's multiple comparison test (GraphPad Prism Software, La Jolla, CA). Differences were considered significant when $p<0.05$ and are indicated as follows: $" p<0.05$; ${ }^{* * *} p<0.005$; ${ }^{* * *} p<0.001$; or N.S., not significant.

\section{Results}

PTP1B is an essential modulator of TDP-43-induced inflammation in astrocytes

To investigate the interaction between TDP-43 and PTP1B in astrocytes, we first sorted GFP-positive cells among Gfp- and GFP-tagged TDP-43-transfected astrocytes and then assessed the levels of PTP1B by immunoblotting. PTP1B expression was significantly increased after TDP-43 transfection in primary astrocytes compared to Gfp-transfected cells (Fig. 1a, b). Furthermore, TDP-43 protein levels were also greatly increased in the insoluble fraction of TDP-43-overexpressing cells (Figure S2a). Lipofectamine-only treatment did not affect the viability of primary astrocytes (Figure S3a). Recent data have shown that PTP1B is a positive regulator of neuroinflammation in microglia [25]. In line with this evidence, we also observed that TDP-43 overexpression in primary astrocytes upregulated the expression of inflammatory genes (Il-1b, Il-6, Lcn2, iNOS, or Nf-kb) (Fig. 1c-l) compared to the levels observed after GFP overexpression in primary astrocytes, further supporting the idea that TDP-43 overexpression induced inflammation in primary astrocytes. To rule out the possibility of off-target effects resulting from the PTP1B inhibitor, we treated cells with an siRNA against the Ptp1b gene. The protein level of PTP1B was markedly decreased by Ptp $1 b$ siRNA 


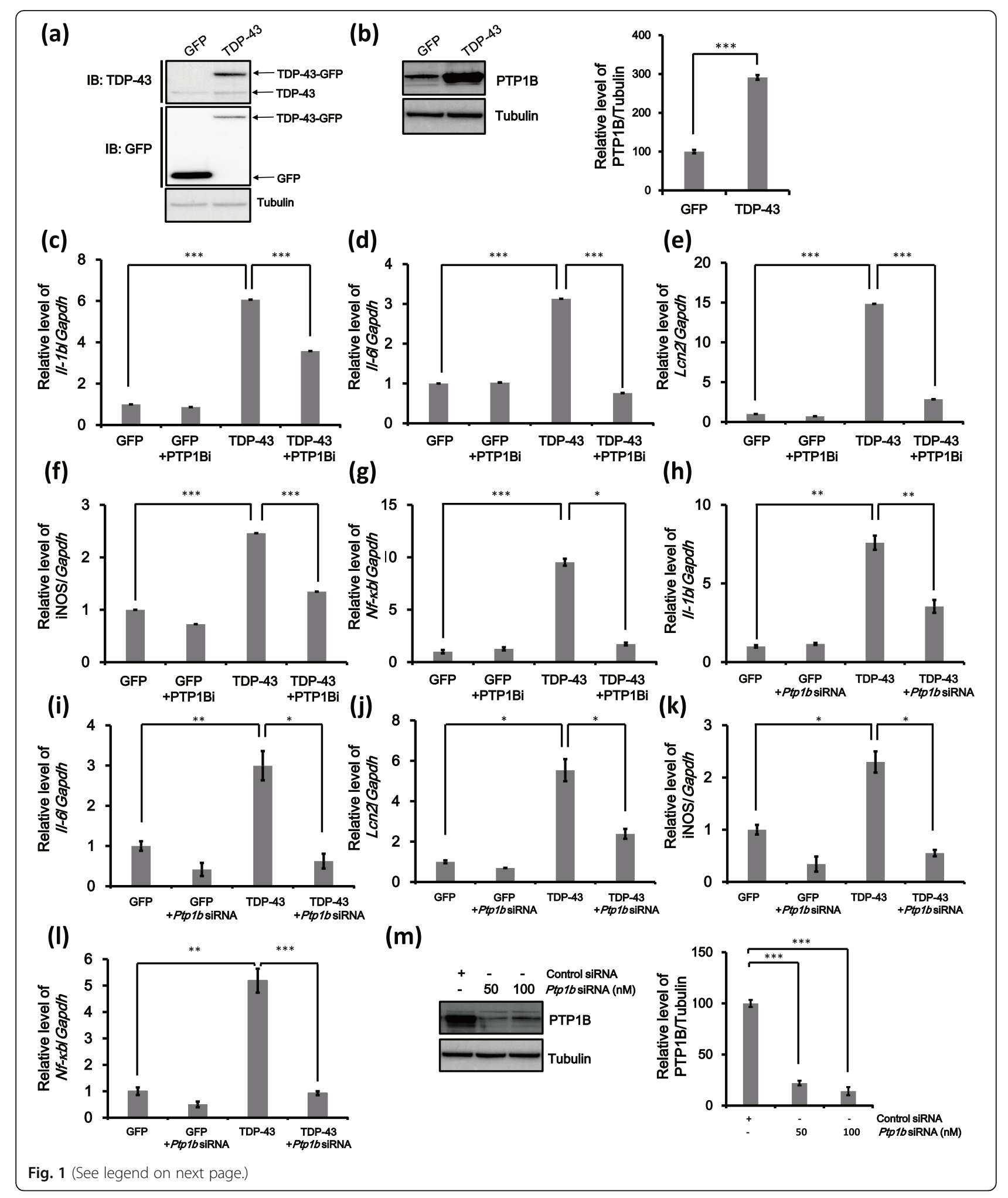




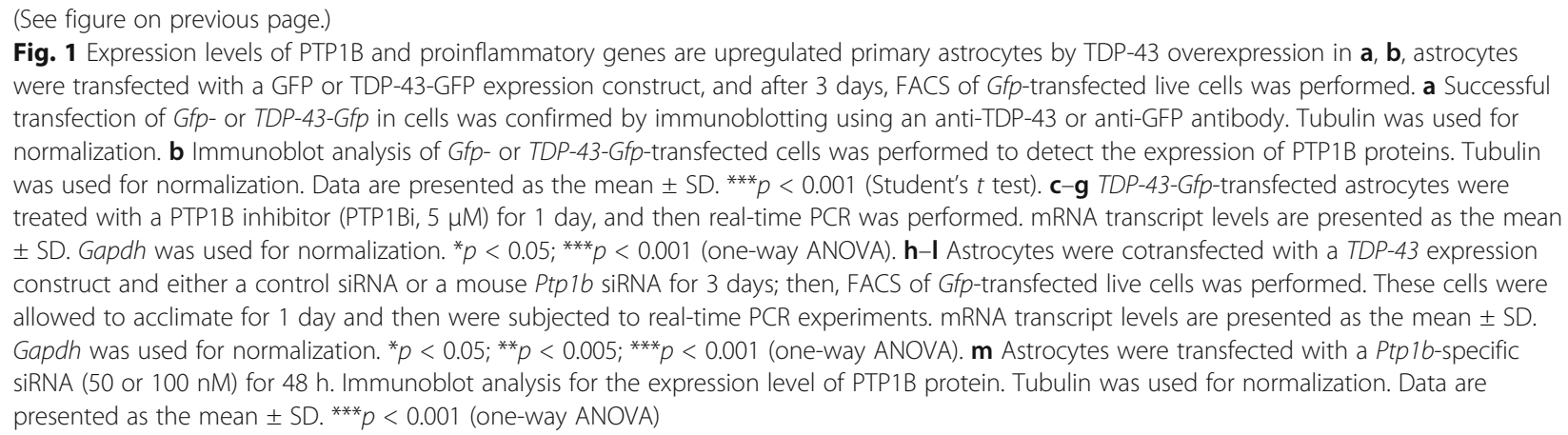

transfection in primary astrocytes (Fig. $1 \mathrm{~m})$. Real-time PCR analysis using Gapdh or $18 S$ rRNA as an internal control indicated that the TDP-43-induced inflammatory gene level was similarly decreased by PTP1B inhibitor treatment and by Ptp $1 b$ siRNA transfection of mouse primary astrocytes (Figs. 1c-l and S4a-j). Taken together, our data demonstrated that TDP-43 overexpression activates the inflammatory response and that PTP1B is a critical regulator of TDP-43-induced neuroinflammation in primary astrocytes.

\section{PTP1B regulates TDP-43-induced inflammation via the NF-KB pathway}

Next, we investigated the mechanism by which PTP1B regulates the TDP-43-induced inflammatory response. A previous study showed that TNF- $\alpha$-induced PTP1B upregulation results in the activation of the NF- $\kappa B$ pathway in rat hypothalamic organotypic cultures [40]. Furthermore, TDP- 43 protein treatment activates microglia via the NF- $k B$ pathway [41]. Thus, we postulated that the NF- $\kappa B$ pathway is also involved in TDP-43-mediated neuroinflammation in astrocytes. To support this hypothesis, we extracted proteins from Gfp- and TDP43-transfected astrocytes and then assessed the phosphorylation level of NF-kB subunit p65 using a phospho-specific (Ser536) anti-p65 antibody. Ser536 phosphorylation of $\mathrm{p} 65$ is required for nuclear translocation of NF- $\mathrm{kB}$, and nuclear translocation of the NF- $\mathrm{kB}$ complex induces expression of inflammatory genes [42]. NF-kB p65 (Ser536) phosphorylation was significantly increased in TDP-43-transfected cells compared to Gfptransfected cells (Fig. 2a). Furthermore, TDP-43-induced NF- $\kappa B$ p65 phosphorylation (Ser536) was restored by PTP1B inhibition (Fig. 2a; $135 \pm 1.7 \%$ in the TDP-43 group, and $93 \pm 2.6 \%$ in the TDP-43 + PTP1Bi group; all test groups were compared to the Gfp transfected astrocyte group). PTP1B inhibition did not affect total NF- $\mathrm{kB}$ protein levels in Gfp- or TDP-43-transfected cells (Fig. 2a).

Next, we performed immunoblotting to determine the effect of PTP1B inhibition on TDP-43 protein levels. The results showed that the increased TDP- 43 protein level in TDP-43-overexpressing cells was not changed by PTP1B inhibition; thus, PTP1B does not seem to directly affect TDP-43 protein levels (Fig. 2b).

Nuclear-cytoplasmic fractionation of primary astrocytes using NE-PER Nuclear and Cytoplasmic Extraction Reagents was employed to determine whether PTP1B regulates the nuclear translocation of $\mathrm{p} 65$ in response to TDP-43. The level of nuclear NF-kB p65 was significantly higher in TDP-43-transfected astrocytes than it was in Gfp-transfected cells, and PTP1B inhibition effectively suppressed the TDP-43-induced nuclear translocation of NF-kB p65 (Fig. 2c $21.5 \pm 1.3 \%$ in the TDP43 group versus $9.3 \pm 1.4 \%$ in the TDP- $43+$ PTP1Bi group; all test groups were compared to the Gfp-transfected astrocyte group).

We also assessed the levels of inflammatory cytokines and chemokines using a cytokine proteome profiler array (blotting) in the ACM obtained from GFP-expressing astrocytes, TDP-43-expressing astrocytes and TDP-43GFP + PTP1Bi-treated astrocytes (data not shown). Notably, we found that the secretion levels of interleukin 1 beta (IL-1 $\beta$ ), interleukin 6 (IL-6) and tumour necrosis factor alpha (TNF- $\alpha$ ) were greatly increased in the TDP43 ACM group compared with the GFP ACM group. Next, we measured the concentrations of IL- $1 \beta$, IL-6 and TNF- $\alpha$ in the ACM using ELISA Development Kits (R\&D Systems; Fig. 2d-f). These 3 proinflammatory cytokines were markedly upregulated in the ACM obtained from TDP-43-overexpressing astrocytes (Fig. 2d-f; IL$1 \beta: 4.50 \pm 0.8 \mathrm{ng} / \mathrm{ml}$ in the GFP ACM group versus 51.7 $\pm 1.5 \mathrm{ng} / \mathrm{ml}$ in the TDP-43 ACM group; IL-6: $17.9 \pm 1.5$ $\mathrm{ng} / \mathrm{ml}$ in the GFP ACM group versus $30.7 \pm 6.0 \mathrm{ng} / \mathrm{ml}$ in the TDP-43 ACM group; and TNF- $\alpha$ : $52.5 \pm 1.4 \mathrm{ng} /$ $\mathrm{ml}$ in the GFP ACM group versus $71.1 \pm 0.2 \mathrm{ng} / \mathrm{ml}$ in the TDP-43 ACM group). Moreover, PTP1B inhibition suppressed the secretion of inflammatory cytokines caused by TDP-43 overexpression (Fig. 2d-f; IL-1 $3: 51.7$ $\pm 1.5 \mathrm{ng} / \mathrm{ml}$ in the TDP-43 ACM group versus $6.6 \pm 0.4$ $\mathrm{ng} / \mathrm{ml}$ in the TDP-43 + PTP1Bi ACM group; IL-6: 30.7 $\pm 6.0 \mathrm{ng} / \mathrm{ml}$ in the TDP-43 ACM group versus $17.3 \pm$ $2.3 \mathrm{ng} / \mathrm{ml}$ in the TDP-43 + PTP1Bi ACM group; and 
(a)
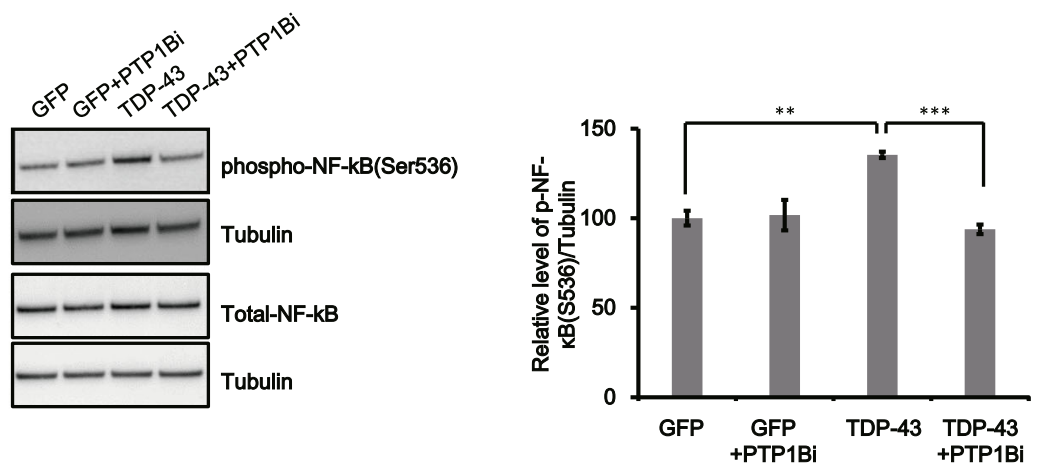

(b)

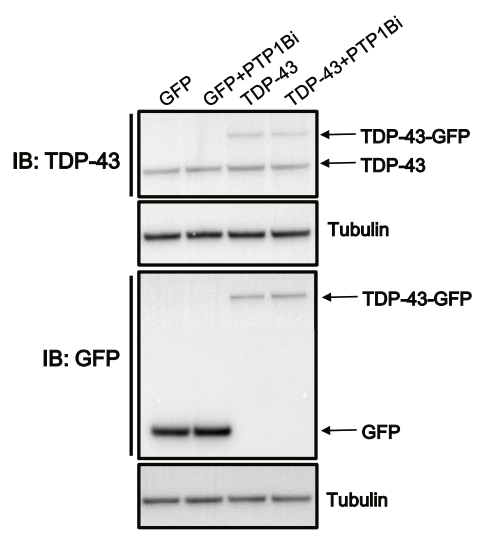

(d)

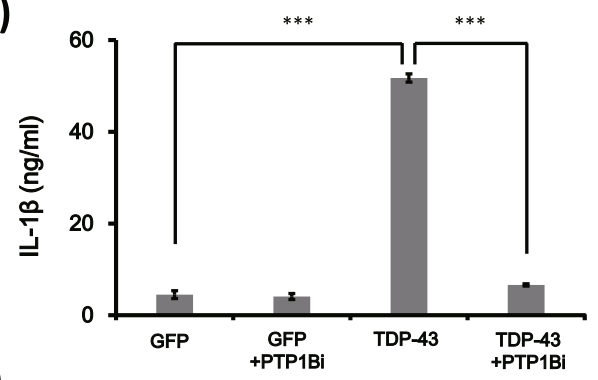

(f)

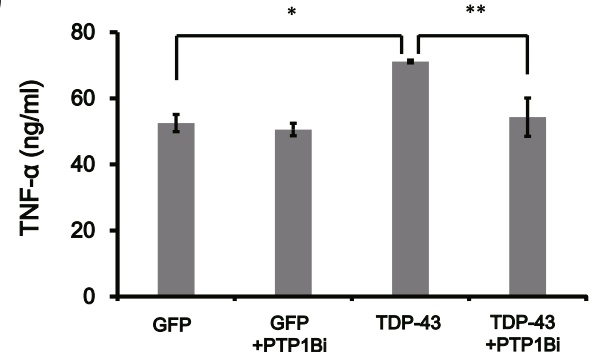

(c)
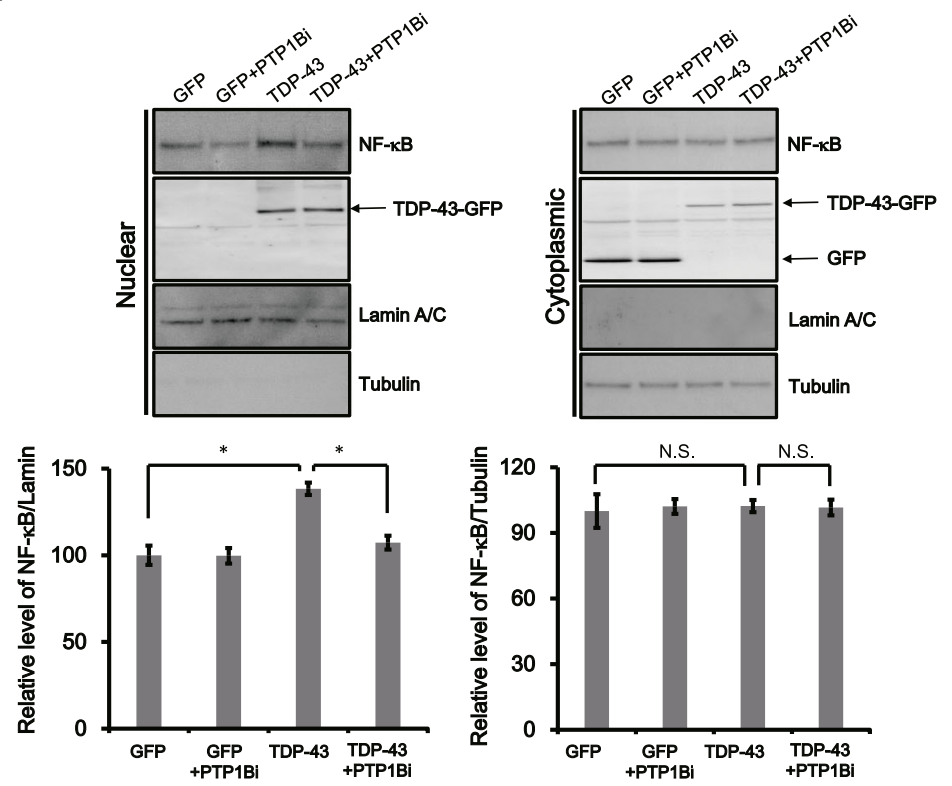

(e)

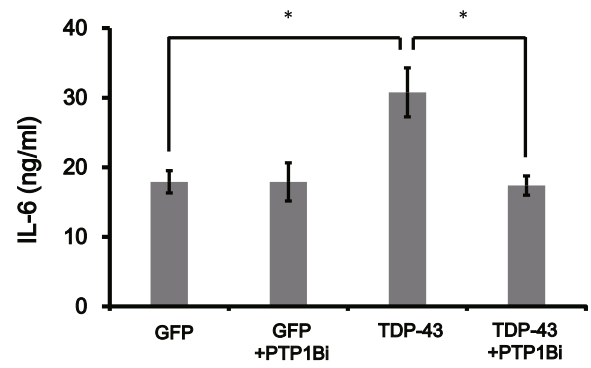

(g)
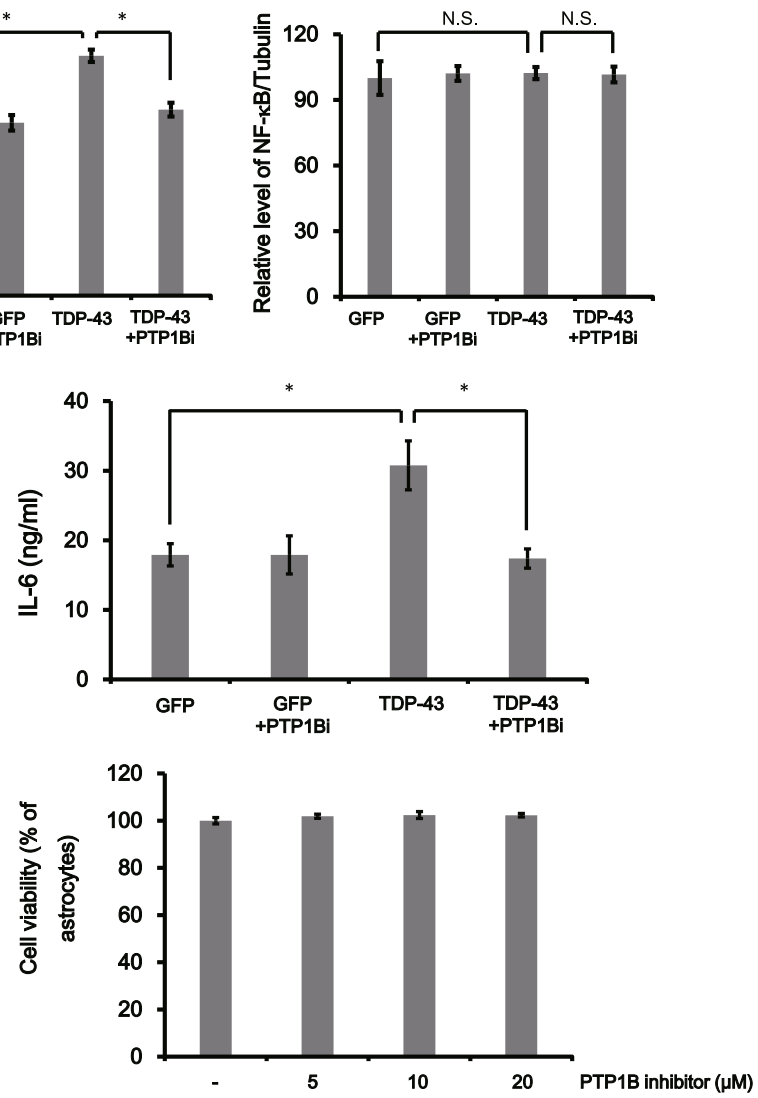

Fig. 2 (See legend on next page.) 
(See figure on previous page.)

Fig. 2 PTP1B inhibition suppresses TDP-43-induced inflammation via the NF-KB pathway. a-c TDP-43-Gfp-transfected astrocytes were treated with PTP1B inhibitor (PTP1Bi, $5 \mu \mathrm{M}$ ) for $24 \mathrm{~h}$ and then were analysed by immunoblotting. a Immunoblot analysis of Gfp- or TDP-43-Gfp-transfected cells was performed to detect the protein expression of phosphorylated NF-kB (Ser536) and total NF-KB after PTP1B inhibitor (PTP1Bi, $5 \mu M$ ) treatment for $24 \mathrm{~h}$. Tubulin was used for normalization. Data are presented as the mean $\pm \mathrm{SD}$. ${ }^{* *} p<0.005$; ${ }^{* * *} p<0.001$ (one-way ANOVA). $\mathbf{b}$ Expression of Gfp- or TDP-43-Gfp in cells was confirmed by immunoblotting using an anti-TDP-43 or anti-GFP antibody. Tubulin was used for normalization. c Cells were fractionated into nuclear and cytoplasmic extracts. Immunoblot analysis was performed for NF-KB protein in the nuclear and cytoplasmic fractions. Lamin A/C (nuclear fraction) and tubulin (cytoplasmic fraction) were used for normalization. Data are presented as the mean \pm SD. ${ }^{*} p<0.05$; N.S. not significant (one-way ANOVA). d-f Gfp- or TDP-43-Gfp-transfected astrocytes were treated with a PTP1B inhibitor (PTP1Bi, $5 \mu \mathrm{M}$ ) for $24 \mathrm{~h}$, and then the ACMs were harvested. ELISAs were conducted for secreted cytokines (IL-1 $\beta$, IL-6 and TNF-a) in the GFP ACM, TDP-43 ACM and TDP-43 + PTP1Bi ACM groups. Data are presented as the mean \pm SD. ${ }^{*} p<0.05 ;{ }^{* *} p<0.005$; and ${ }^{* * *} p<0.001$ (one-way ANOVA). $g$ A CCK-8 assays were performed to assess the viability of primary astrocytes treated with the PTP1B inhibitor (PTP1Bi, $5 \mu \mathrm{M}, 10 \mu \mathrm{M}$ or $20 \mu \mathrm{M})$ for $24 \mathrm{~h}$. Data are presented as the mean \pm SD

TNF- $\alpha$ : $71.1 \pm 0.2 \mathrm{ng} / \mathrm{ml}$ in the TDP-43 ACM group versus $54.3 \pm 3.3 \mathrm{ng} / \mathrm{ml}$ in the TDP-43 + PTP1Bi ACM group). We also showed that the PTP1B inhibitor alone did not affect the viability of astrocytes (Fig. $2 \mathrm{~g}$ ). These data suggest that PTP1B inhibition mitigates TDP-43induced inflammation via the NF- $\mathrm{KB}$ pathway in astrocytes.

\section{PTP1B inhibition and absorption of proinflammatory cytokines mitigate the neuronal toxicity caused by astrocytic TDP-43 overexpression}

To examine the effect of PTP1B on astrocytic TDP-43induced neuronal toxicity, we used neuron-astrocyte transwell cocultures and ACM-treated neuron culture. Overexpression of TDP-43 in primary astrocytes caused neuronal toxicity in transwell cultures (Fig. 3a, b). This neuronal toxicity was significantly mitigated by PTP1B inhibitor treatment or Ptp $1 b$ siRNA transfection (Fig. 3b; $77 \pm 5.1 \%$ in the TDP-43 group versus $94 \pm 1.2 \%$ in the TDP-43 + PTP1Bi group, and all test groups were compared to the Gfp-transfected + DMSO-treated group; 79 $\pm 2.5 \%$ in the TDP-43-GFP + control siRNA group versus $90 \pm 3.0 \%$ in the TDP-43-GFP + Ptp1b siRNA group, and all test groups were compared to the Gfp + control siRNA cotransfected group). We also showed that the PTP1B inhibitor alone did not affect neuronal viability. Similar to the results of the transwell culture assays, the ACM from TDP-43-overexpressing astrocytes with PTP1B inhibited showed lower neurotoxicity than the TDP-43 ACM in primary cortical neuron culture (Fig. 3d; $73 \pm 0.5 \%$ in the TDP-43 ACM versus $87 \pm$ $0.7 \%$ in the TDP-43-GFP + PTP1Bi ACM group, and all test groups were compared to Gfp-transfected + DMSOtreated ACM group; $82 \pm 1.2 \%$ in the TDP-43-GFP + control siRNA ACM group versus $90 \pm 0.3 \%$ in the TDP-43-GFP + Ptp1b siRNA ACM group, and all test groups were compared to Gfp + control siRNA cotransfected ACM group). We also confirmed the CCK- 8 assay data by staining with the fluorescent cell tracker (green) CMFDA. The number of CMFDA-positive neurons was notably decreased in cocultures with TDP-43-Gfp- transfected astrocytes $(68 \pm 8.6 \%)$, and PTP1B downregulation significantly attenuated astrocytic TDP-43induced neuronal death (Fig. 3e; $83 \pm 5.8 \%$ ). On the other hand, Ptp1b knockdown in astrocytes did not affect the viability of neuronal cells in the neuronastrocyte transwell and ACM cocultures. Moreover, the ACM obtained from PTP1B inhibitor-treated astrocytes did not affect neuronal viability (Figure S5a).

We next investigated whether proinflammatory cytokines regulate neuronal toxicity under astrocytic TDP-43 overexpression. Our data showed that treatment with anti-IL-1 $\beta$ (50 ng/ml), anti-IL-6 $(50 \mathrm{ng} / \mathrm{ml})$ and antiTNF- $\alpha(100 \mathrm{ng} / \mathrm{ml})$ neutralizing antibodies suppressed TDP-43-induced neuronal toxicity. The concentration of the neutralizing antibodies used in the treatment was determined with reference to previous experiments [43$45]$. The presence of an antibody targeting IL- $1 \beta$, IL- 6 or TNF- $\alpha$ dramatically attenuated neuronal toxicity treated with TDP-43 ACM in primary cortical neurons (Fig. 3f; IL-1 $\beta$ : $54.6 \pm 0.3 \%$ in the TDP-43 ACM group versus $70.2 \pm 6.4 \%$ in the TDP- 43 ACM + anti-IL- $1 \beta$ antibody group; IL-6: $54.6 \pm 0.3 \%$ in the TDP-43 ACM group versus $70.1 \pm 1.8 \%$ in the TDP-43 ACM + anti-IL-6 antibody group; TNF- $\alpha$ : $54.6 \pm 0.3 \%$ in the TDP- 43 ACM group versus $67.8 \pm 0.6 \%$ in the TDP- $43 \mathrm{ACM}+$ antiTNF- $\alpha$ antibody group; all test groups were compared to the GFP ACM group). A control IgG antibody did not affect neuronal viability (Fig. 3g; $77.8 \pm 1.3 \%$ in the TDP-43 ACM group versus $79.5 \pm 1.8 \%$ in the TDP-43 $\mathrm{ACM}+$ control IgG antibody group; all test groups were compared to the GFP ACM group). To confirm these results, neurons were labelled with CMFDA dye, which functioned as a fluorescent cell tracker. Consistently, TDP-43 ACM treatment also decreased the number of CMFDA-positive cells, and treatment with a bioactive antibody targeting IL-1 $\beta$, IL- 6 or TNF- $\alpha$ significantly attenuated TDP-43 ACM-induced neuronal toxicity (Fig. 3h; IL-1 1 : $52.6 \pm 2.1 \%$ in the TDP-43 ACM group versus $80.6 \pm 1.4 \%$ in the TDP-43 ACM + anti-IL-1 $\beta$ antibody group; IL-6: $52.6 \pm 2.1 \%$ in the TDP-43 ACM group versus $78.5 \pm 1.5 \%$ in the TDP- 


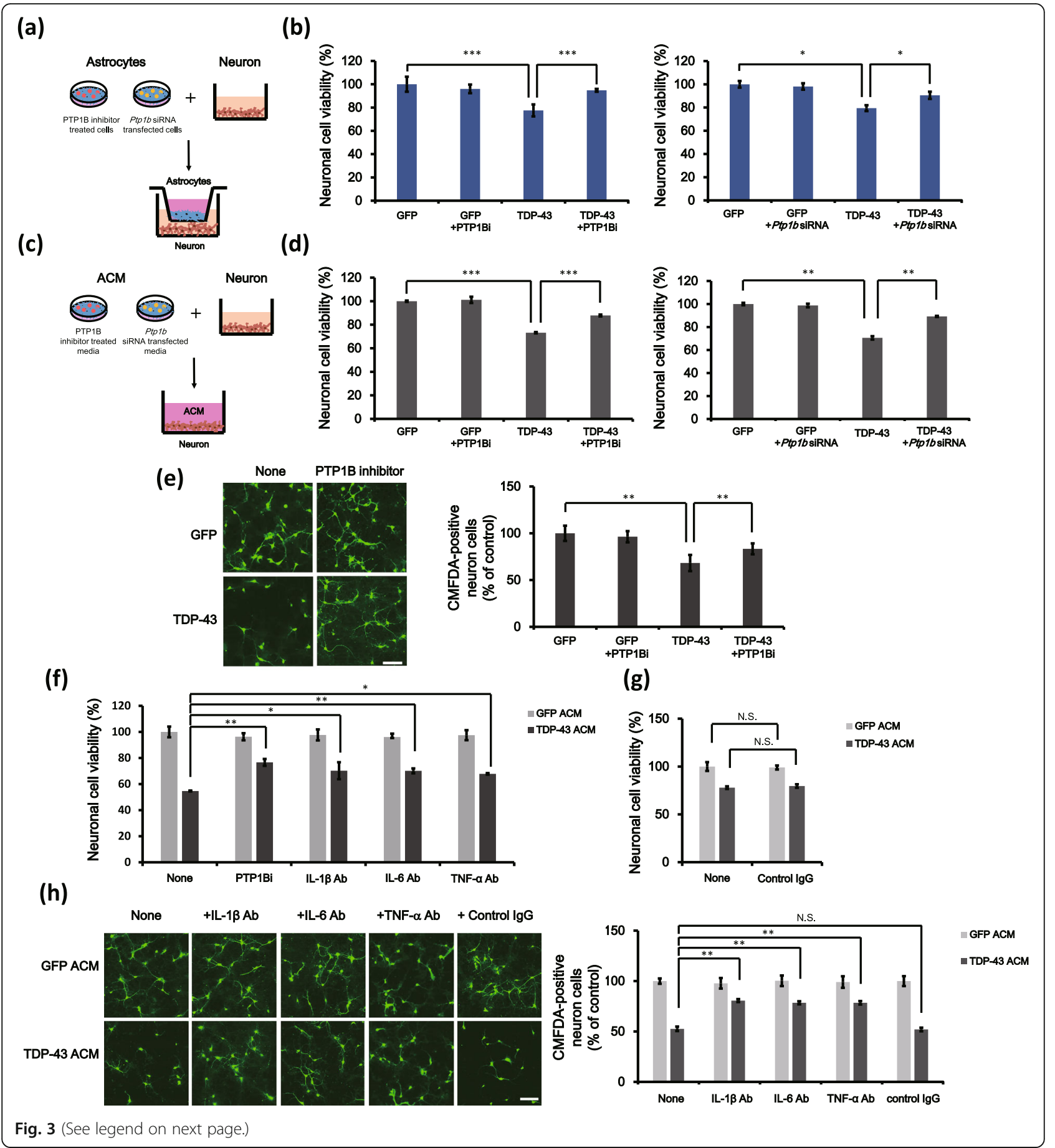


(See figure on previous page.)

Fig. 3 PTP1B inhibition and absorption of proinflammatory cytokines mitigate neuronal toxicity caused by TDP-43 overexpression in astrocytes. a, b Neuron-astrocyte coculture. b TDP-43-Gfp-transfected astrocytes were treated with a PTP1B inhibitor (PTP1Bi, $5 \mu$ M) or Ptp1b siRNA (50 nM) for $24 \mathrm{~h}$ and then were cocultured with primary cortical neurons in transwell culture inserts. Neuronal viability was measured using a CCK-8 assay after a coculture period of 36 or $48 \mathrm{~h}$. Data are presented as the mean \pm SD. ${ }^{*} p<0.05 ;{ }^{* *} p<0.001$ (one-way ANOVA). c-e ACM-treated neuron culture. $\mathbf{d}$ TDP-43-Gfp-transfected astrocytes were treated with a PTP1B inhibitor (PTP1Bi, $5 \mu \mathrm{M}$ ) or Ptp 1b siRNA (50 nM) for $24 \mathrm{~h}$, and then the ACM were harvested. Primary cortical neurons were stimulated with GFP ACM, TDP-43 ACM or TDP-43 + PTP1Bi ACM for 5 days, and then a CCK8 assay was performed. Data are presented as the mean \pm SD. ${ }^{* *} p<0.005 ;{ }^{* * *} p<0.001$ (one-way ANOVA). e At the end of neuron-ACM coculture, primary cortical neurons were stained with CMFDA (green). Then, CMFDA-positive neurons were counted under a fluorescence microscope. Data are presented as the mean \pm SD of $3 .{ }^{* *} p<0.005$ (one-way ANOVA). Scale bars, $20 \mu \mathrm{m}$. $\mathbf{f}-\mathbf{h}$ Primary cortical neurons stimulated with TDP-43 ACM were treated with IL-1 $\beta$ antibody $(50 \mathrm{ng} / \mathrm{ml})$, IL-6 antibody $(50 \mathrm{ng} / \mathrm{ml})$ and TNF-a antibody $(10 \mathrm{ng} / \mathrm{ml})$ for 5 days and then were subjected to a CCK-8 assay or to CMFDA staining. $\mathbf{f}$ CCK-8 assays were performed to assess the viability of primary cortical neurons. Data are presented as the mean $\pm \mathrm{SD}$. ${ }^{*} p<0.05 ;{ }^{* *} p<0.005$ (one-way ANOVA). $\mathbf{g}$ CCK-8 assays were performed to assess the viability of primary cortical neurons stimulated with TDP-43-transfected ACM and treated with a control lgG antibody $(50 \mathrm{ng} / \mathrm{ml}$ ) for 5 days. Data are presented as the mean \pm SD. N.S. not significant (Student's $t$ test). $\mathbf{h}$ CMFDA-positive neurons were counted under a fluorescence microscope. Data are presented as the mean \pm SD. ${ }^{* *} p<0.005$; N.S. not significant (one-way ANOVA). Scale bars, $20 \mu \mathrm{m}$

$43 \mathrm{ACM}+$ anti-IL-6 antibody group; TNF- $\alpha$ : $52.6 \pm$ $2.1 \%$ in the TDP-43 ACM group versus $78.8 \pm 1.4 \%$ in the TDP-43 ACM + anti-TNF- $\alpha$ antibody group; all test groups were compared to the GFP ACM group). Moreover, GFP and GFP + PTP1Bi ACM supplemented with IL-1 $\beta$, IL- 6 and TNF- $\alpha$ proteins also resulted in neurotoxicity similar to that of TDP-43 ACM (Figure S6a; $100 \pm 2.0 \%$ in the GFP ACM group versus $66 \pm 3.0 \%$ in the GFP ACM + IL- $1 \beta+$ IL-6 + TNF- $\alpha$ protein group; $99 \pm 2.3 \%$ in the GFP + PTP1Bi ACM group versus $63 \pm 2.0 \%$ in the GFP + PTP1Bi ACM + IL-1 $\beta+$ IL-6 + TNF- $\alpha$ protein group). Furthermore, siRNA knockdown of $I l-1 b, I l-6$ and Tnf- $\alpha$ in TDP-43-overexpressing astrocytes dramatically attenuated TDP-43-induced neuronal toxicity and reduced secretion of proinflammatory cytokines (Figure S7a-d). These data indicate that the upregulation of proinflammatory cytokines such as IL-1 $\beta$, IL- 6 and TNF- $\alpha$ is an essential process in astrocytic TDP-43-induced neuronal toxicity.

\section{PTP1B inhibition suppresses astrocytic TDP-43-induced mitochondrial dysfunction and spine retraction in neurons}

Recent studies have suggested that mitochondrial dysfunction is a critical factor for many neurodegenerative diseases, including AD, PD and ALS [46-48]. Moreover, previous studies have indicated that TDP-43 is linked to mitochondrial dysfunction and abnormalities of mitochondrial dynamics [49-52]. Therefore, TDP-43induced mitochondrial defects could be a key feature of disease pathology. To investigate whether TDP-43 overexpression in astrocytes could induce mitochondrial dysfunction in neurons, we measured the cellular OCR using a Seahorse XF24 Extracellular Flux Analyzer and a mitochondrial stress test kit (Seahorse Bioscience) in ACM-treated primary cortical neurons. The OCR is an indicator of mitochondrial respiration. OCR measurement in cultured neurons using electron transport chain-regulating agents such as oligomycin, FCCP and rotenone/antimycin A allows for the analysis of mitochondrial respiratory parameters, including basal respiration, ATP production, maximal respiration and spare respiratory capacity. The basal mitochondrial respiration was not significantly affected by TDP-43 ACM treatment (Fig. 4a, b; $86.4 \pm 6.0 \%$ in the TDP-43 ACM group versus $92.7 \pm 5.0 \%$ in the TDP-43+ PTP1Bi ACM group; all test groups were compared to the Gfp-transfected + DMSO-treated ACM group). However, ATP production, maximal respiration and spare respiratory capacity were markedly decreased by TDP-43 ACM treatment in primary cortical neurons (Fig. 4a, b). Consistent with the cell viability assay results, TDP-43induced neuronal mitochondrial dysfunction in astrocytes was greatly ameliorated by PTP1B inhibition (Fig. $4 \mathrm{a}, \mathrm{b}$, ATP production: $65.6 \pm 6.8 \%$ in the TDP-43 ACM group versus $94.6 \pm 8.2 \%$ in the TDP-43 + PTP1Bi ACM group; maximal respiration capacity: $63.9 \pm 6.6 \%$ in the TDP-43 ACM group versus $92.4 \pm 8.5 \%$ in the TDP-43 + PTP1Bi ACM group; spare respiratory capacity: $40.8 \pm$ $7.7 \%$ in the TDP-43 ACM group versus $85.8 \pm 13.8 \%$ in the TDP-43 + PTP1Bi ACM group; all test groups were compared to the Gfp-transfected + DMSO-treated ACM group). Additionally, OCR values were normalized for each group according to total cellular protein concentration (Fig. 4c).

Mitochondrial functions associate with various neuronal diseases and locally modulate the formation of growth cones to affect the direction and rate of neurite growth $[49,53,54]$. Moreover, TDP-43 affects the local translation of dendritic mRNAs, which contributes to synaptic plasticity [55]. Therefore, we analysed neuronal morphology changes by TDP-43-induced neurodegeneration in astrocytes. TDP-43 ACMtreated neurons showed a significant reduction in spine density and length compared to those of GFP ACM-treated neurons (Fig. 4d-f). Quantitative analysis data of spine density and length were 


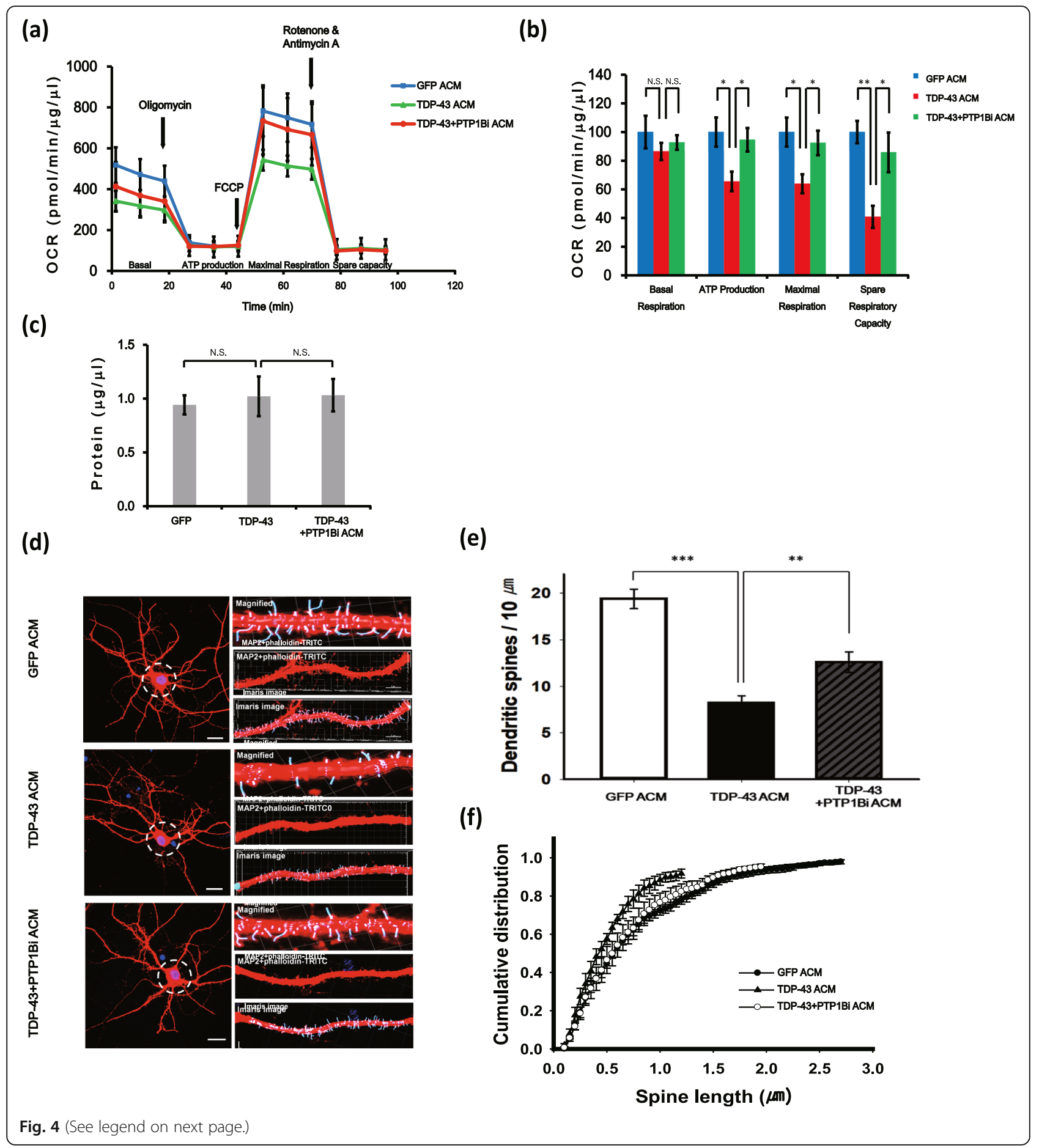


(See figure on previous page.)

Fig. 4 PTP1B inhibition suppresses TDP-43-induced mitochondrial dysfunction in astrocytes and spine retraction in primary cortical neurons. a-c Primary cortical neurons in XF24-well culture plates were stimulated with GFP ACM, TDP-43 ACM or TDP-43 + PTP1Bi ACM for 5 days. a Analysis of mitochondrial dysfunction in ACM-treated cells was performed by a Seahorse XF analyser to detect the basal OCR, ATP production, maximum reserve and respiratory capacity. The OCR was normalized to the total protein concentration (OD). $\mathbf{b}$ Quantification of the OCR, ATP production, maximum reserve, and respiratory capacity as a percentage of the basal values. Data are presented as the mean \pm SEM. ${ }^{*} p<0.05$; ${ }^{* *} p<0.005$; and N.S. not significant (one-way ANOVA). c The protein concentrations of cell lysates in each ACM-treated group were measured by a BCA protein assay kit. Data are presented as the mean \pm SD. N.S. not significant (one-way ANOVA). $\mathbf{d}$ Representative confocal images of each ACMtreated group. Dendritic spines in the dotted circle, which is $20 \mu \mathrm{m}$ radius from the centre of nucleus, were not analysed (panel left). Scale bars, $20 \mathrm{\mu m}$. Representative confocal (phalloidin-TRITC, red and anti-Map2-Alexa 594, red) and filament tracing Imaris images from randomly selected dendritic spines of each ACM-treated neuron group (right panel). Scale bars, $5 \mu \mathrm{m}$. The magnified image in the right panels shows filament tracing of the analysed spine. Scale bars, $2 \mu \mathrm{m}$. e Dendritic spine density was defined as the number of spines per $50 \mu \mathrm{m}$ of dendrite length in the Imaris image of the right panel of Fig. $4 \mathrm{~d}$, which shows the average number of dendritic spines for $10 \mu \mathrm{m}$. Fifteen dendrites were randomly selected from four separate cortical neuron cultures per GFP ACM, TDP-43 ACM and TDP-43+PTP1Bi ACM-treated cortical neuron group. ${ }^{* *} p<$ 0.005 , *** $p 0.001$ (one-way ANOVA). $\mathbf{f}$ Cumulative distribution curves of spine length from cortical neurons as determined by the KolmogorovSmirnov (KS) test: mean spine length of GFP ACM vs. TDP-43 ACM and TDP-43 ACM vs. TDP-43 + PTP1Bi ACM. $p=0.027$ and $p=$ 0.609 , respectively

significantly decreased by treatment with the TDP-43 ACM compared to the GFP ACM in cortical neurons, whereas both spine density and length were significantly recovered in the TDP-43+ PTP1Bi ACM group compared to TDP-43 ACM group (Fig. 4d-f). Taken together, these data indicate that PTP1B inhibition mitigates several neurodegenerative phenotypes caused by TDP-43-induced alterations in astrocytes.

\section{PTP1B inhibition ameliorates astrocytic TDP-43-induced} neuronal toxicity and mitochondrial dysfunction in motor neuron-like cells

We next investigated whether PTP1B inhibition also mitigates astrocytic TDP-43-induced neuronal toxicity in motor neuron-like cells. To do this, we induced the differentiation process in NSC-34 cells. Previous studies indicate that differentiated NSC-34 cells show motor neuron-like properties, such as neurite extension and the expression of specific motor neuron markers $[34,56$, 57]. Differentiated NSC-34 cells showed motor neuronlike morphology and markedly increased transcription of motor neuron markers Map2, Mapt, Gap43, chAT and AchE (Fig. 5a, b). Similar to the results of the primary cortical neurons, the cellular toxicity of TDP-43 ACM in differentiated NSC-34 cells was significantly attenuated by PTP1B inhibition (Fig. 5c; $80 \pm 3.6 \%$ in the TDP- 43 ACM group versus $90 \pm 1.3 \%$ in the TDP- $43+$ PTP1Bi ACM group; all test groups were compared to the Gfptransfected + DMSO-treated ACM group). In addition, treatment with antibodies targeting IL-1 $\beta$, IL- 6 and TNF- $\alpha$ dramatically attenuated the cellular toxicity of TDP-43 ACM in differentiated NSC-34 cells (Fig. 5d; 69 $\pm 1.7 \%$ in the TDP- 43 ACM group versus $90 \pm 1.6 \%$ in the TDP-43 ACM + anti-IL-1 $\beta$ + anti-IL-6 + anti-TNF$\alpha$ antibody group; all test groups were compared to the GFP ACM group).

To further confirm that IL-1 $\beta$, IL- 6 and TNF- $\alpha$ proteins are essential components of astrocytic TDP-43- induced neurotoxicity, the effect of IL- $1 \beta$, IL- 6 and TNF- $\alpha$ treatment was examined in differentiated NSC34 cells. Similar to the situation observed with TDP-43 ACM treatment, GFP or GFP + PTP1Bi ACM supplemented IL-1 $\beta$, IL- 6 and TNF- $\alpha$ caused neurotoxicity (Figure S6b; $100 \pm 3.1 \%$ in the GFP ACM group versus $44 \pm 2.0 \%$ in the GFP ACM + IL- $1 \beta+$ IL- $6+$ TNF- $\alpha$ protein group; $98 \pm 1.1 \%$ in the GFP + PTP1Bi ACM group versus $46 \pm 0.7 \%$ in the GFP + PTP1Bi ACM + IL-1 $\beta+$ IL- $6+$ TNF- $\alpha$ protein group).

To investigate whether TDP-43 overexpression in astrocytes could induce mitochondrial dysfunction in motor neuron-like cells, we measured the cellular OCR in ACM-treated differentiated NSC-34 cells. The basal mitochondrial respiration, ATP production, maximal respiration and spare respiratory capacity were markedly decreased by TDP-43 ACM treatment in differentiated NSC-34 cells (Fig. 5e, f). Similar to the results observed in primary cortical neurons, TDP-43-induced mitochondrial dysfunction in astrocytes was greatly mitigated by PTP1B inhibition in differentiated NSC-34 cells (Fig. 5e, f; basal mitochondrial respiration: $67.9 \pm 4.7 \%$ in the TDP-43 ACM group versus $87.4 \pm 3.9 \%$ in the TDP-43 + PTP1Bi ACM group; ATP production: $51.2 \pm 4.4 \%$ in the TDP-43 ACM group versus $67.8 \pm 3.4 \%$ in the TDP-43 + PTP1Bi ACM group; maximal respiration capacity: $113.8 \pm 2.5 \%$ in the TDP- $43 \mathrm{ACM}$ group versus $161.2 \pm 7.8 \%$ in the TDP-43 + PTP1Bi ACM group; spare respiratory capacity: $34.2 \pm 0.9 \%$ in the TDP-43 ACM group versus $48.9 \pm 2.7 \%$ in the TDP43 + PTP1Bi ACM group; all test groups were compared to Gfp-transfected + DMSO-treated ACM group). Moreover, GFP ACM or GFP + PTP1Bi ACM supplemented with IL-1 $\beta$, IL- 6 and TNF- $\alpha$ caused mitochondrial dysfunction in differentiated NSC-34 cells (Figure S6c). These results suggest that PTP1B also regulates TDP-43-induced astrocyte death in motor neuron-like cells. 
(a)

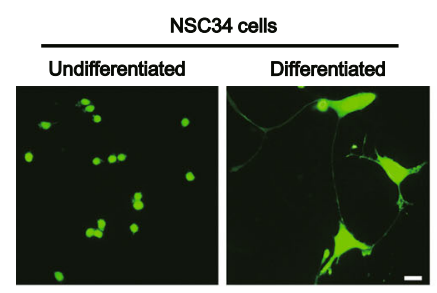

(c)

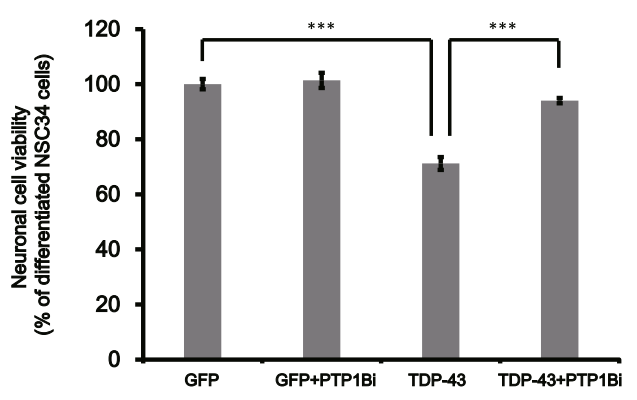

(b)

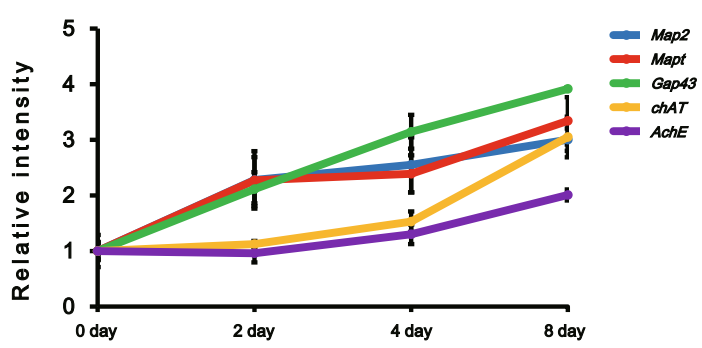

(d)

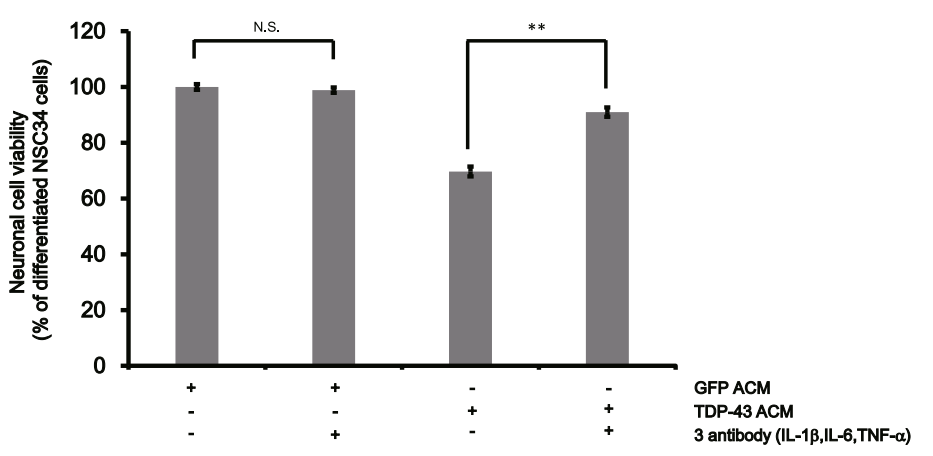

(e)

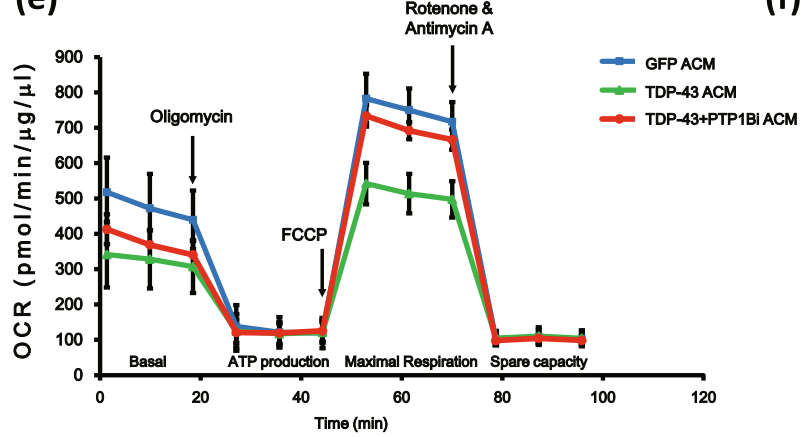

(f)

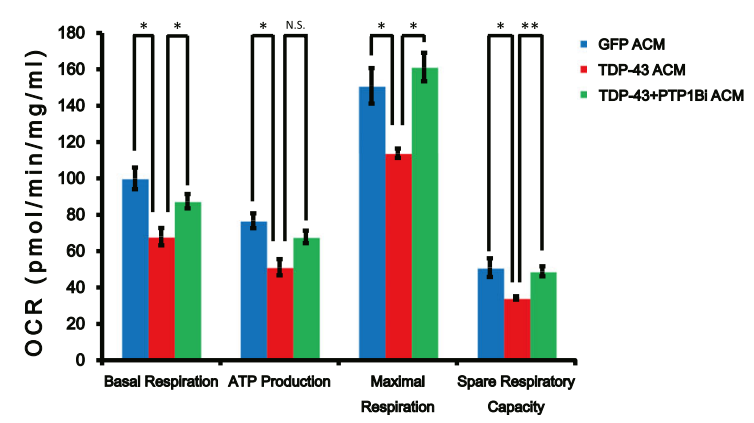

Fig. 5 PTP1B inhibition attenuates astrocytic TDP-43-induced toxicity and mitochondrial dysfunction in differentiated NSC-34 motor neuron-like cells. a-f NSC-34 cells were treated with $1 \mu \mathrm{M}$ RA for 8 days and then were analysed. a CMFDA staining of undifferentiated or differentiated NSC-34 cells at 8 days. Scale bars, $100 \mu \mathrm{m}$. b Real-time PCR results of motor neuronal marker genes in undifferentiated or differentiated NSC-34 cells at the indicated time points. Data are presented as the mean \pm SD. Gapdh was used for normalization. $\mathbf{c}$ Differentiated NSC-34 cells were stimulated with GFP ACM, TDP-43 ACM or TDP-43+ PTP1Bi ACM for 4 days. Cell viability was analysed by CCK-8 assay. ${ }^{* * *} p<0.005$ (one-way ANOVA). d Differentiated NSC-34 cells stimulated with TDP-43 ACM were treated with an IL-1 $\beta$ antibody $(50 \mathrm{ng} / \mathrm{ml})$, an IL-6 antibody $(50 \mathrm{ng} / \mathrm{ml})$ and a TNF-a antibody $(100 \mathrm{ng} / \mathrm{ml})$ for 4 days and then were subjected to CCK-8 assays. TDP-43 ACM-induced toxicity was rescued by IL-1 $\beta, I L-6$ and TNF-a antibody treatment. Data are presented as the mean \pm SD. ${ }^{*} p<0.05$; ${ }^{* *} p<0.005$ (one-way ANOVA). e, $\mathbf{f}$ Differentiated NSC-34 cells in XF24-well culture plates were stimulated with GFP ACM, TDP-43 ACM or TDP-43 + PTP1Bi ACM for 4 days. e Mitochondrial dysfunction analysis of ACM-treated cells was performed with a Seahorse XF analyser to detect the basal OCR, ATP production, maximum reserve and respiratory capacity. The OCR was normalized to total protein concentration. $\mathbf{f}$ Quantification of the OCR, ATP production, maximum reserve and respiratory capacity as a percentage of the basal values. Data are presented as the mean \pm SEM. ${ }^{*} p<0.05$; ${ }^{* *} p<0.005$; and N.S. not significant (one-way ANOVA)

Inflammation and neuronal toxicity induced by glial TDP-43 are mitigated by Ptp1b downregulation in Drosophila

Previous studies indicate that the NF- $\kappa B$ pathway is a key mechanism for regulating inflammation in Drosophila [58]. We wondered whether the modification of PTP1B expression affected TDP-43-induced inflammation in vivo, so we used a Drosophila ALS model that expressed human TDP-43 in all glial cells. Overexpression of TDP-43 in glial cells significantly increased Dorsal $(N f-\kappa b)$ and $i N O S$ levels after 5 days (Fig. 6a, b). The downregulation of Ptp $1 b$ in Drosophila glial cells suppressed the levels of inflammatory genes induced by TDP-43 expression (Fig. 6a, b) without changing the expression level of TDP-43 (Fig. 6e). The knockdown 


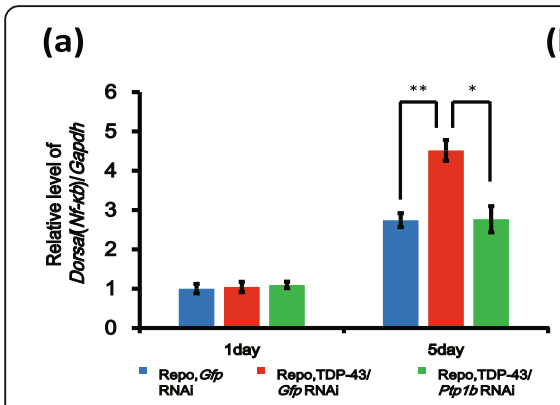

(d)

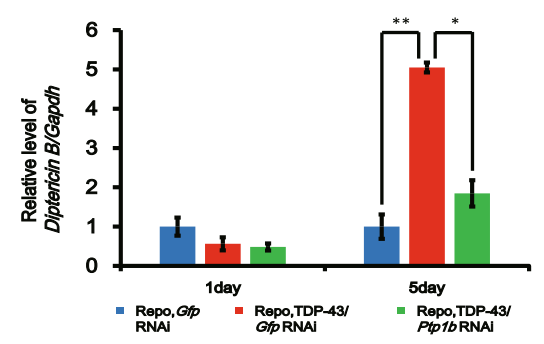

(g)

(b)

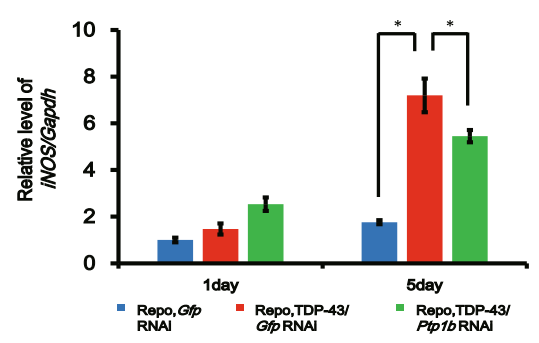

(e) (c)

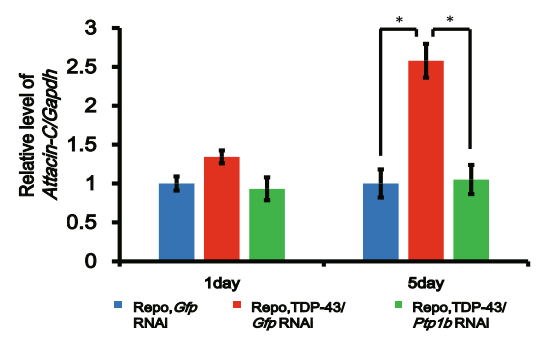

(f)
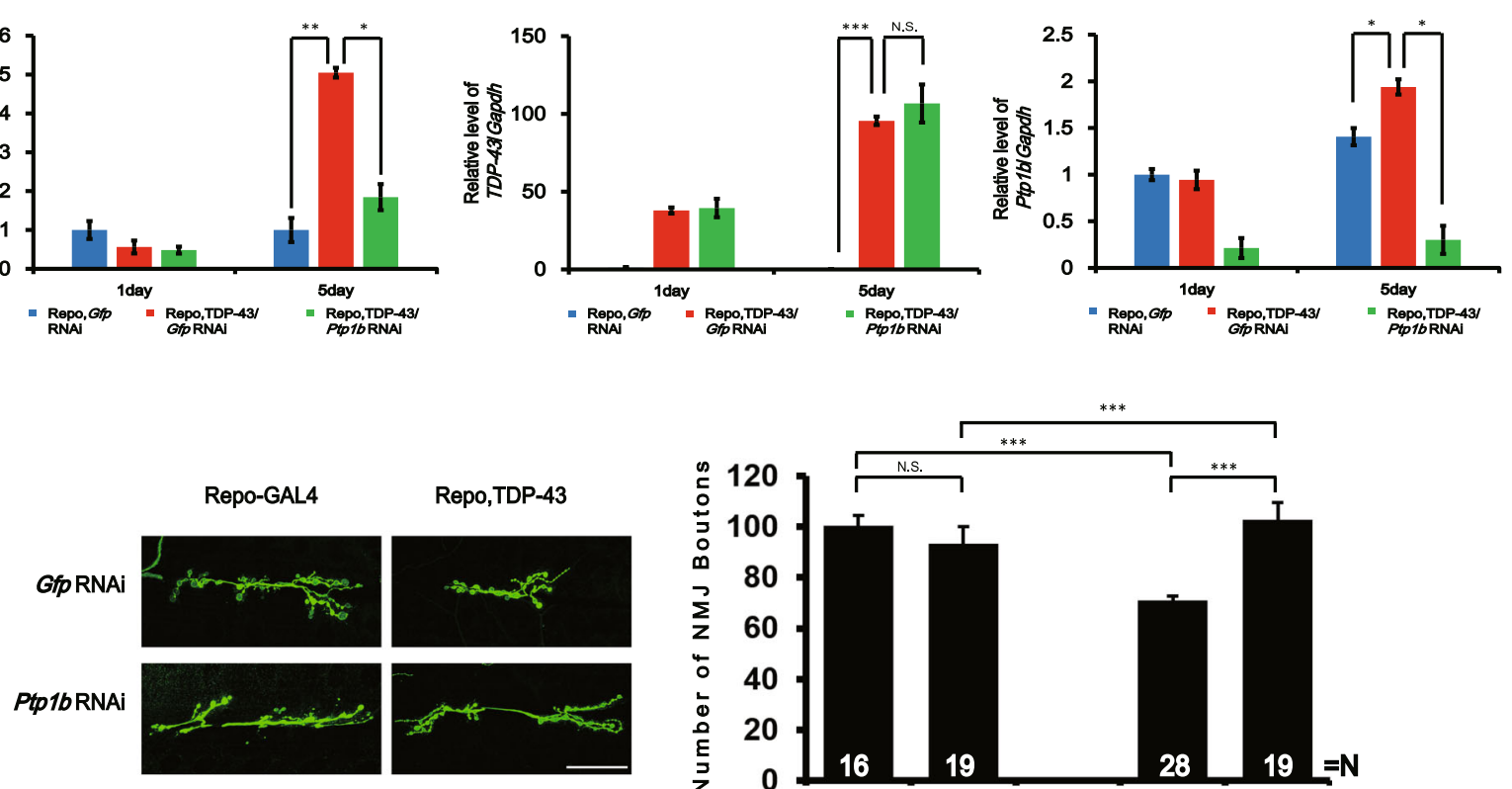

(f)
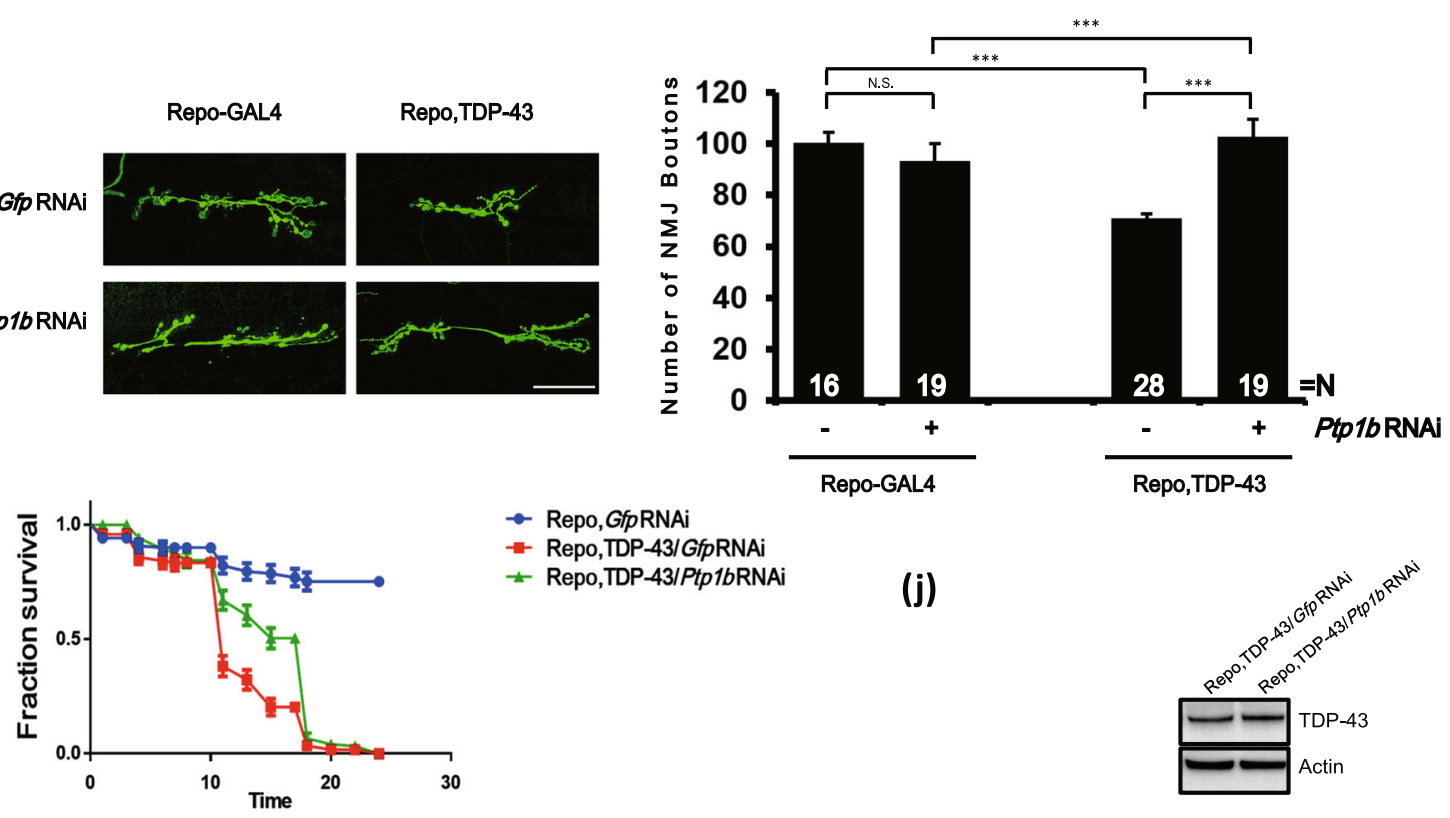

- Repo,GfpRNAi

- Repo,TDP-43/GfpRNAi

- Repo,TDP-43/Ptp1bRNAi

(j)

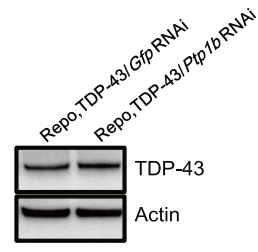

(i)
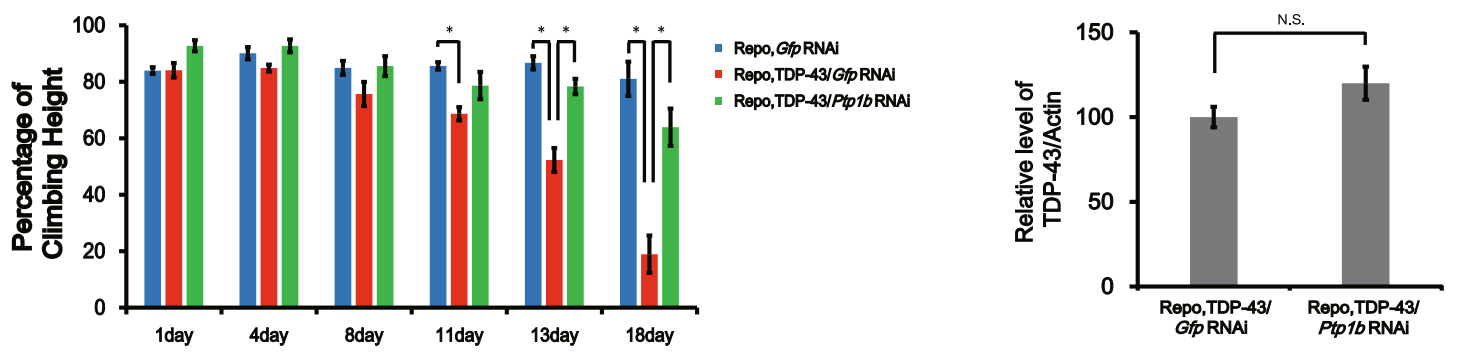

Fig. 6 (See legend on next page.) 


\section{(See figure on previous page.)}

Fig. 6 The inflammation and neuronal toxicity effects induced by glial TDP-43 are mitigated by Ptp61f downregulation in Drosophila. a, b Levels of Dorsal (Nf-kb) and iNOS mRNA from fly head lysates of control or TDP-43 glial-expressing transgenic flies were analysed by real-time PCR. Gapdh was used for normalization. Data are presented as the mean \pm SD. ${ }^{*} p<0.05 ;{ }^{* *} p<0.005$ (one-way ANOVA). $\mathbf{c}$, d Levels of Attacin-C and Diptericin B mRNA from fly head lysates of control or TDP-43-expressing glial transgenic flies were analysed by real-time PCR. Gapdh was used for normalization. Data are presented as the mean \pm SD. ${ }^{*} p<0.05 ;{ }^{* *} p<0.005$ (one-way ANOVA). e, $\mathbf{f}$ Levels of Tdp-43 and Ptp 16 mRNA from fly head lysates of control or TDP-43-expressing glial transgenic flies were analysed by real-time PCR. Gapdh was used for normalization. Data are presented as the mean $\pm \mathrm{SD} .{ }^{*} p<0.05 ;{ }^{* * *} p<0.001$; N.S. not significant (one-way ANOVA). $\mathbf{g}$ Immunostaining of third instar larval NMJs under wild-type (Repo-Gal4/+) and TDP-43-overexpressing (Repo-Gal4/TDP-43) conditions. Labelling of NMJs to determine their morphology in larva was conducted with anti-horseradish peroxidase (green). Scale bars, $50 \mu \mathrm{m}$. Data are presented as the mean \pm SEM. ${ }^{* * *} p<0.001 ; N . S$. not significant (one-way ANOVA). $\mathbf{h}$ Survival rate of flies treated with Repo-Gal4/GFP RNAi, Repo,TDP-43/GFP RNAi or Repo,TDP-43/PTP1B RNAi at the indicated time points. Data are presented as the mean \pm SEM. i Climbing activity of flies treated with Repo-Gal4/GFP RNAi, Repo, TDP-43/GFP RNAi or Repo,TDP-43/PTP1B RNAi at the indicated time points. Data are presented as the mean \pm SEM. ${ }^{*} p<0.05$ (one-way ANOVA). $\mathbf{j}$ Immunoblot analysis of TDP-43 proteins from head lysates of control or TDP-43 glial transgenic flies. Actin was used for normalization. Data are presented as the mean \pm SD. N.S. not significant (Student's $t$ test). $\mathbf{a}-\mathbf{j}$ Genotypes: GFP is Repo-Gal4/UAS-Egfp RNAi VALIUM20-EGFP.shRNA.4, GFP + PTP1B RNAi is Repo-Gal4/UAS-Ptp61f RNAi HMS00421, TDP-43 is Repo-Gal4/UAS-TDP-43/UAS-Egfp RNAiVALIUM20-EGFP.shRNA.4 and TDP-43+PTP1B RNAi is RepoGal4/UAS-TDP-43/UAS-Ptp61f RNAi HMS00427

efficiency of Ptp61f RNAi (the Drosophila homologue of PTP1B) was high, as Ptp61f levels were low (Fig. 6f). In addition, the activation of NF-kB leads to the production of antimicrobial peptides (AMPs), such as Attacin, Diptericin and Cecropin [59-61]. We examined the effects of Ptp1b and TDP-43 expression on TDP-43-induced AMP genes (Figs. 6c, d and S8a-f). TDP-43-expressing flies showed markedly increased Attacin-C and Diptericin $B$ expression. Attacin- $C$ and diptericin $B$ expression was significantly suppressed by the downregulation of Ptp1b in Drosophila (Figs. 6c, d and S8a-f). These results suggest that the downregulation of $P t p 1 b$ in the Drosophila glial system is sufficient for attenuating TDP43-induced inflammation.

To investigate the relevance of PTP1B for TDP-43induced neurotoxicity, we used a model of Drosophila expressing human TDP-43 in all types of glial cells, including astrocyte-like glia. Although TDP-43 can induce inflammation, it is still not clear whether the inhibition of PTP1B is implicated in TDP-43mediated neurotoxicity in Drosophila. The Drosophila NMJ is a powerful biological system for studying synaptic defects in neurodegenerative diseases (Lu et al. 2011; Lee et al. 2012; McGurk et al. 2015). A recent study showed that the overexpression of TDP-43 in motor neurons leads to a significant disruption of NMJ morphology (Coyne et al. 2014). In another study, it was shown that disrupted expression of TDP-43 at NMJs impairs BMP signalling (Deshpande et al. 2016). However, glial toxicity caused by TDP- 43 has not been well-characterized in NMJs. We found that glial expression of TDP-43 at larval NMJs resulted in an $\sim 30 \%$ reduction in the bouton number. This effect was rescued by knocking down Ptp61f expression with an RNAi (Fig. 6g). These results provide strong evidence for a genetic role of PTP1B in regulating neuronal toxicity caused by TDP-43 overexpression in glia.
Previously, we revealed that flies expressing TDP-43 showed a markedly reduced lifespan and climbing ability compared to those of controls [11]. We also examined the effects of Ptp1b and TDP-43 expression on TDP-43induced lifespan reduction and climbing dysfunction. The TDP-43-expressing flies showed a markedly reduced lifespan and climbing ability (Fig. 6h, i) without any change in the expression level of TDP-43 (Fig. 6j). The lifespan and climbing defects induced by TDP- 43 were significantly rescued by the downregulation of Ptp61f in Drosophila (Fig. 6h, i). At 18 days of age, flies expressing TDP43 in their glia showed a markedly reduced climbing ability compared to control animals $(18.9 \pm 6.6 \%$ in the Repo, TDP-43/GFP RNAi versus $81.0 \pm 6.0 \%$ in the Repo-Gal4/ GFP RNAi). This climbing deficit was greatly attenuated by knockdown of Ptp61f (18.9 $\pm 6.6 \%$ in the Repo,TDP43/GFP RNAi versus $63.9 \pm 6.5 \%$ in the Repo,TDP-43/ PTP1B RNAi), with the flies also exhibiting a recovered motility. Knockdown of Ptp61f alone had no effect on climbing ability or lifespan (data not shown). These results indicate that PTP1B inhibition mitigates neuronal toxicity caused by pan-glial TDP-43-induced inflammation in flies.

Taken together, our data support a model whereby TDP-43 in astrocytes causes neurodegeneration at least in part by inducing the inflammatory response via PTP1B (Fig. 7). We found that TDP-43-induced PTP1B upregulation eventually leads to activation of the NF- $\mathrm{kB}$ pathway in astrocytes. Activation of the NF- $\mathrm{KB}$ pathway also increases the secretion of inflammatory cytokines, such as IL- $1 \beta$, IL- 6 and TNF- $\alpha$. Consequently, proinflammatory activation of astrocytes induces mitochondrial dysfunction, spine retraction, and cell death in nearby neurons.

\section{Discussion}

We demonstrated for the first time that PTP1B is a major regulator of the TDP-43-induced inflammatory response in astrocytes. It is already known that the TDP-43 protein initiates the proinflammatory cascade in 


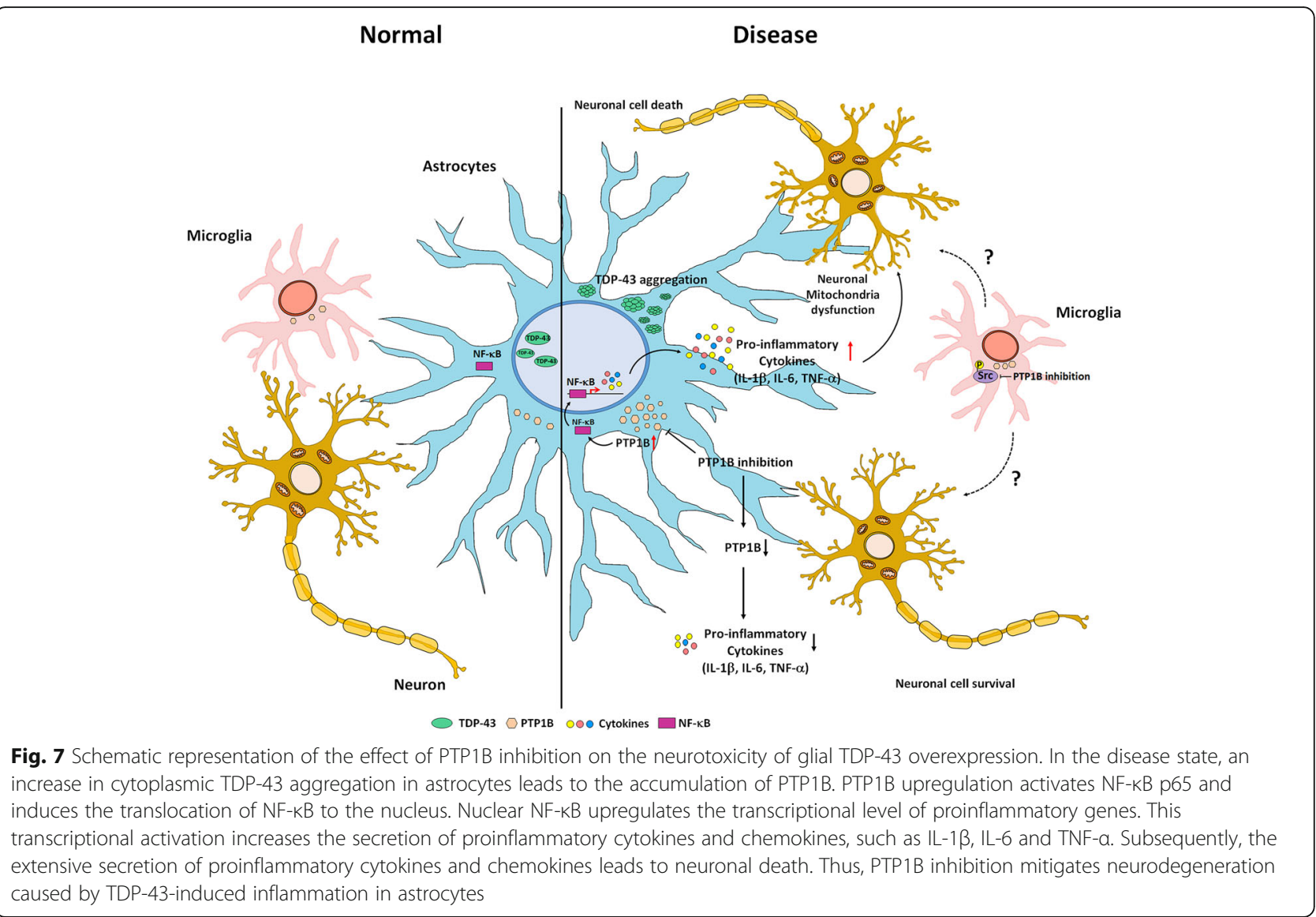

microglia [41, 62]; however, its role in astrocytes was previously unknown. Our present study shows changes in the inflammatory and toxic functions of astrocytes due to TDP-43, and it provides a mechanistic understanding of the role of these alterations in exacerbating neurodegeneration.

Previous studies have shown that astrocytes isolated from ALS patients are toxic to mouse embryonic stem cell-derived motor neurons in coculture [63]. More recently, Qian et al. demonstrated that astrocytes differentiated from induced pluripotent stem cells of ALS patients cause movement deficits and neurodegeneration in mice following their transplantation into the spinal cords of SCID mice [64]. Although the underlying molecular mechanism of astrocyte-mediated neurotoxicity in ALS is not well understood, accumulating evidence suggests that astrocytes are implicated in neurodegeneration in ALS.

Recent studies have demonstrated that PTP1B positively modulates neuroinflammation via the NF- $\mathrm{KB}$ pathway in microglia $[25,65]$. However, the role of PTP1B in astrocytes is largely unknown. Here, we found that PTP1B inhibition effectively attenuates the TDP-43induced inflammatory response in astrocytes. Similar to what is observed in microglia, PTP1B regulates the inflammatory response via the NF- $\mathrm{kB}$ pathway (Fig. $2 \mathrm{a}-\mathrm{c}$ ).
NF- $\kappa \mathrm{B}$ activation is observed in the astrocytes of ALS patients [66]. In addition, Kia et al. showed that astrocytes expressing ALS-linked mutant FUS were toxic to motor neurons and that mutant FUS-expressing astrocyte-induced neurotoxicity is mediated by the NF$\kappa B$ pathway [45]. Notably, TDP-43 directly binds to the p65 subunit of NF- $\mathrm{kB}$ and acts as a coactivator of the NF- $\mathrm{BB}$ pathway in BV-2 microglial cells [67]. These findings suggest that activation of NF- $\mathrm{kB}$ in astrocytes is implicated in neurodegeneration in ALS and that this activation might be induced by TDP-43 accumulation.

The secretion of proinflammatory cytokines is known to be associated with glia-mediated neurotoxicity. Previous studies have found that administration of IL- $1 \beta$ in the rat brain dramatically enhances neuronal damage induced by ischaemia and excitotoxicity $[68,69]$. In this study, we found that the secretion of IL- $1 \beta$, IL- 6 and TNF- $\alpha$ in astrocytes was elevated by overexpression of TDP-43 (Fig. 2d-f). Interestingly, these cytokines were previously shown to be significantly elevated in the peripheral blood of ALS patients [70]. Moreover, a recent meta-analysis revealed that TNF- $\alpha$ was significantly increased in the cerebrospinal fluid (CSF) of ALS patients [71]. To determine whether the increase in inflammatory cytokine levels by astrocytic TDP-43 is related to 
neurotoxicity, we treated cells with neutralizing antibodies targeting IL-1 $\beta$, IL- 6 and TNF- $\alpha$. Accordingly, we found that TDP-43-overexpressing astrocyte-induced neurotoxicity is attenuated by treatment with these neutralizing antibodies (Fig. 3f-h). Our findings, together with others, suggest that TDP-43 upregulation in astrocytes may be linked to elevated levels of proinflammatory cytokines and that these cytokines are mediators of neuronal death in ALS.

Mitochondrial dysfunction in affected neurons is a common feature of ALS [72]. However, although numerous studies have shown that neuronal TDP-43 accumulation causes abnormalities in mitochondrial morphology, dynamics and function with in vivo and in vitro models $[8$, 52, 73-75], it is unknown whether the accumulation of TDP-43 in glia can induce neuronal mitochondrial impairment. In this study, seahorse analysis of mitochondrial bioenergetics in cortical neurons treated with TDP-43 ACM revealed that TDP-43 ACM significantly impaired the maximal respiration rate and the ATP-linked respiration rate. Moreover, PTP1B inhibition greatly attenuated TDP-43 ACM-induced mitochondrial dysfunction (Fig. 4a, b). Collectively, the findings from our study demonstrated that TDP-43 accumulation in astrocytes drives neuronal mitochondrial defects via the PTP1B-mediated inflammatory response. To study the effects of TDP-43 on motor neuron-like cell lines, we investigated the toxicity and mitochondrial dysfunction induced by TDP-43 in differentiated NSC-34 cells. NSC-34 is a hybrid cell line produced by the fusion of neuroblastoma/spinal cord neurons, and it is often used as a bona fide cellular model to investigate the physiological mechanism of ALS [76]. Our data confirmed that PTP1B inhibition mitigated cytotoxicity and mitochondrial dysfunction induced by TDP43 ACM in differentiated NCS-34 cells (Fig. 5c-f).

NF-kB-mediated immune responses in glia are well conserved in Drosophila [77]. Furthermore, in Drosophila, genetic suppression of the NF-kB pathway (Imd/Relish) greatly attenuated the neuronal TDP-43-induced shortening of lifespan [78]. Although some studies indicate that glial TDP-43 expression causes premature death, motility deficits and larval NMJ defects [79-81], it is still unclear whether glial TDP-43 can induce neuroinflammation in Drosophila. In this study, our data in Drosophila showed that the expression of inflammatory genes (Dorsal $(N f-\kappa b)$, $i N O S$, Attacin-C, Diptericin B) is greatly increased by panglial TDP-43 expression (Fig. 6a-d). Importantly, our data also showed that genetic knockdown of fly PTP1B effectively restored the upregulation of those inflammatory genes. Consistent with previous results, pan-glial expression of TDP-43 in Drosophila causes larval NMJ defects, a shortened lifespan and climbing defects, and these pathologic phenotypes were significantly suppressed by knockdown of fly PTP1B (Fig. 6g-i). These findings suggest that inhibition of PTP1B mitigates glial TDP-43-induced neurotoxicity in vivo.

Three glial cell types are mainly associated with major neurons in the Drosophila central nervous system (CNS): astrocyte-like, ensheathing and cortex glia. These 3 types of glia share the function of mammalian astrocytes. They surround neuronal cell bodies and proximal neurites, are coupled to the vasculature, and associate closely with synapses [82]. To regulate target genes in all glial cells, we used the repo-GAL4 driver, which expresses GAL4 protein in all types of glial cells except for midline glia. Similar to the results of mammalian astrocytes, glial TDP-43-induced inflammation and neurotoxicity are significantly attenuated by knockdown of Ptp $1 b$. Thus, we believe that the fly model data using repoGAL4 further confirm the results from the mouse astrocyte primary culture experiments. However, we cannot rule out the possibility that the functions of fly glial cells are different from those of mouse astrocytes, which may have affected these results. Thus, further in-depth studies are warranted to elucidate how PTP1B regulates glial TDP-43-induced neurodegeneration in Drosophila.

\section{Conclusion}

Our data highlight the therapeutic potential of PTP1B in treatment of ALS. Since PTP1B is a well-known therapeutic target for diabetes and obesity, numerous PTP1B inhibitors have already been developed [83]. Therefore, analysing the effects of the previously developed PTP1B inhibitors on astrocytic TDP-43-induced neurotoxicity may help to identify promising therapeutic agents.

\section{Supplementary information}

Supplementary information accompanies this paper at https://doi.org/10. 1186/s12974-020-01963-6.

Additional file 1: Figure S1. Purity of cultured primary mouse cortica neurons and astrocytes. (a) Primary cortical neuron (upper) or astrocyte (lower) enriched cultures were stained with antibodies for neuron, astrocyte and microglia markers. Triple immunostaining of MAP2 (neuron; red), GFAP (astrocytes; green), and Iba-1 (microglia; pink) in primary neuronal cells at DIV 7 and astrocytes at DIV 21. DAPI staining was used to determine the number of cells. Scale bar, $200 \mu \mathrm{m}$. $n=248$ cells (primary cortical neuron culture), $n=447$ cells (primary astrocyte culture). Figure S2. Insoluble TDP-43 protein was significantly increased in TDP-43overexpressing primary astrocytes. (a) Immunoblot analysis of TDP-43 protein in the insoluble and soluble fractions of TDP-43-Gfp-transfected astrocytes. The immunoblot results from 3 independent experiments were normalized to those of tubulin. Figure S3. Transfection of the control plasmid did not affect the viability of astrocytes. (a) Astrocytes were treated with the Lipofectamine only or with a GFP expression DNA vector + Lipofectamine mixture for 3 days; then, CCK-8 assays were performed. Data are presented as the mean \pm SD of 3 independent experiments. N.S., not significant (Student's t-test). Figure S4. PTP1B and proinflammatory genes are upregulated by TDP-43 overexpression in primary astrocytes. (a-e) TDP-43-Gfp-transfected astrocytes were treated with a PTP1B inhibitor (PTP1Bi, $5 \mu \mathrm{M}$ ) for 1 day, and then real-time PCR was performed. 185 rRNA was used as a normalization gene for real-time PCR data. PTP1B inhibition greatly attenuated TDP-43-induced inflammatory upregulation. 
Quantification data for II-1b (a), II-6 (b), Lcn2 (c), iNos (d), and Nf-kb. (e) Quantification data are presented as the mean \pm SD from 3 independent real-time PCR experiments. ${ }^{*} p<0.05 ;{ }^{* *} p<0.005$; and ${ }^{* * *} p<0.001$ (one-way ANOVA with Bonferroni's multiple comparison test). (f-j) Astrocytes were cotransfected with TDP-43 expression construct and a control siRNA or mouse Ptp 16 siRNA for 3 days, and then FACS of Gfp-transfected live cells was performed. These cells were allowed to acclimate for 1 day and then were subjected to real-time PCR experiments. The TDP-43-induced upregulation of inflammatory gene transcription was attenuated by PTP1B downregulation. 185 rRNA was used as a normalization gene for RT-PCR Quantification data for $11-1 b(f), \|-6(\mathrm{~g})$, Lcn2 (h), iNos (i), and Nf-kb. (j) All data are presented as the mean \pm SD from 3 independent real-time PCR experiments. ${ }^{*} p<0.05$; ${ }^{* *} p<0.005$ (one-way ANOVA with Bonferroni's multiple comparison test). Figure $\mathbf{S 5}$. ACM from astrocytes treated with a PTP1B inhibitor does not affect the viability of mouse cortical neurons. (a) Primary cortical neurons were treated with DMSO ACM or PTP1Bi ACM for 5 days and then were subjected to CMFDA staining. CMFDA-positive neurons were counted under a fluorescence microscope. The percentage of CMFDA-positive cells was quantified (lower). Data are presented as the mean \pm SD of 3 independent experiments. N.S., not significant (Student's $t$-test). Scale bars, $20 \mu \mathrm{m}$. Figure S6. The secretion of proinflammatory cytokines such as IL-1 $\beta, I L-6$, and TNF-a mediates astrocytic TDP-43induced neuronal toxicity and mitochondrial dysfunction. Primary cortical neurons (a) and differentiated NSC-34 motor neurons (b) were stimulated with GFP ACM or GFP + PTP1Bi ACM supplemented with IL-1 $\beta$ protein $(50 \mathrm{ng} / \mathrm{ml})$, IL-6 protein $(50 \mathrm{ng} / \mathrm{ml})$, and TNF-a protein $(10 \mathrm{ng} / \mathrm{ml})$ for 4 days, and then they were subjected to CCK-8 assays. Similar to what was observed with TDP-43 ACM treatment, GFP or GFP + PTP1Bi ACM supplemented with IL-1 $\beta, I L-6$, and TNF-a caused neurotoxicity. Data are presented as the mean \pm SD of 3 independent experiments. ${ }^{* *} p<0.005$ (Student's t-test). (c) Differentiated NSC-34 motor neurons stimulated with GFP ACM or GFP + PTP1Bi ACM supplemented with IL-1 $\beta$ protein (50 ng/ $\mathrm{ml})$, IL-6 protein $(50 \mathrm{ng} / \mathrm{ml})$, and TNF-a protein $(10 \mathrm{ng} / \mathrm{ml})$ for 4 days and then subjected to mitochondrial dysfunction analysis. Mitochondrial dysfunction analysis of ACM-treated cells was assessed through the detection of basal OCR, ATP production, maximum reserve and respiratory capacity by a Seahorse XF analyser. The oxygen consumption rate (OCR) was normalized to the total protein concentration (OD). Quantification of the OCR, ATP production, maximum reserve and respiratory capacity as a percentage of the basal values. Data are presented as the mean \pm SEM of 3 independent experiments. ${ }^{*} p<0.05 ;{ }^{* *} p<0.005$; and ${ }^{* * *} p<0.001$ (one-way ANOVA with Bonferroni's multiple comparison test). Figure S7. Knockdown of $\|-1 b\|-$,6 , and Tnf-a mitigated astrocytic TDP-43-induced neuronal toxicity. (a-c) Astrocytes were cotransfected with the TDP-43-Gfp expression construct and control siRNA, II-1b siRNA, II-6 siRNA, or Thf-a siRNA, and after 3 days the ACM were harvested. The concentrations of secreted cytokines (IL-1 $\beta, \mathrm{IL}-6$, and TNF- $\alpha$ ) in the GFP ACM and TDP-43 ACM groups were measured by ELISA. TDP-43-induced secretion of cytokines (IL-1 $\beta$, IL-6, and TNF-a) was significantly suppressed by the downregulation of IL-1 $\beta$ (a), IL-6 (b), or TNF- $a$. (c). Data are presented as the mean \pm SD. ${ }^{*} p<0.05 ;{ }^{* *} p<0.005$; and ${ }^{* * *} p<0.001$ (one-way ANOVA with Bonferroni's multiple comparison test). (d) Primary cortical neurons stimulated with TDP-43ACM from astrocytes were treated with II-16 siRNA, II-6 siRNA, and Tnf-a siRNA for 5 days, and then they were subjected to CCK8 assays. TDP-43 ACM-induced toxicity was rescued by siRNA knockdown of proinflammatory cytokine genes. Data are presented as the mean \pm SD of 3 independent experiments. ${ }^{*} p<0.05 ;{ }^{* *} p<0.005$; and ${ }^{* * *} p<0.001$ (one-way ANOVA with Bonferroni's multiple comparison test). Figure $\mathbf{S 8}$. Inflammation induced by glial TDP-43 is mitigated by Drosophila Ptp 16 downregulation. (a-f) Levels of Dorsal (Nf-kb), iNos, Attacin-C, Diptericin B, TDP-43 and Ptp 16 mRNA from fly head lysates of control or TDP-43expressing glial transgenic flies were analysed by real-time PCR. 185 rRNA was used as a normalization gene for real-time PCR. TDP-43-induced expression of genes involved in inflammation and genes that are downstream of NF-KB was significantly suppressed by the downregulation of PTP1B. Quantification data of Dorsal (Nf-kb) (a), iNos (b), Attacin-C (c), Diptericin B (d), TDP-43 (e), and Ptp1b (f) mRNA transcript levels are presented as the mean \pm SD from 3 independent real-time PCR experiments. $18 \mathrm{~S}$ rRNA was used for normalization. ${ }^{*} p<0.05$; ${ }^{* *} p<0.005$; and ${ }^{* * *} p<0.001$ (one-way ANOVA with Bonferroni's multiple comparison test).

\section{Abbreviations}

ALS: Amyotrophic lateral sclerosis; TDP-43: Transactive response DNA binding protein of 43kDa; FTLD-TDP: Frontotemporal lobar degeneration with TDP43; hnRNP: Heterogeneous nuclear ribonucleoprotein; WT: Wild type; AD: Alzheimer's disease; FTLD: Frontotemporal lobal degeneration; PTP1B: Protein-tyrosine phosphatase 1B; DMSO: Dimethyl sulfoxide; RA: Alltrans retinoic acid; ACM: Astrocyte-conditioned media; CMFDA: Chloromethylfluorescein diacetate; IL-1 $\beta$ : Interleukin 1 beta; IL6: Interleukin 6; TNF-a: Tumour necrosis factor alpha; FCCP: Carbonyl cyanide 4-(trifluoromethoxy) phenylhydrazone; OCR: Oxygen consumption rate; AMPs: Antimicrobial peptides; NMJ: Neuromuscular junction; CSF: Cerebrospinal fluid; CNS: Central nervous system

\section{Acknowledgements}

Not applicable.

\section{Authors' contributions}

SL, SK, H-YK, HRL YK, MJ, Y-MJ and CMH planned and performed the experiments. SRK and KK and provided ideas for the project and participated in writing the paper. SL, SK, SL and H-JK wrote the paper. All authors read and approved the final manuscript.

\section{Funding}

This work was supported by the KBRI Research Program of the Ministry of Science, ICT \& Future Planning (20-BR-02-08); the Soonchunhyang University Research Fund; the Basic Science Research Program through the National Research Foundation of Korea (NRF), funded by the Ministry of Science, ICT \& Future Planning (NRF-2020R1A2C4002366, NRF-2019R1F1A1045639, and NRF2020R1F1A1058459); and the Korea Health Technology R\&D Project through the Korea Health Industry Development Institute (KHIDI), funded by the Ministry of Health and Welfare, South Korea (grant number: HI14C1135 and HI18C1241). Confocal microscopy data were acquired at Research Division and Brain Research Core Facilities in KBRI.

\section{Availability of data and materials}

The authors declare the data and material availability in this manuscript.

\section{Ethics approval and consent to participate}

This study was approved by the Institutional Animal Care and Use Committee (IACUC) at Korea Brain Research Institute (\# IACUC-18-00019). All experiments were performed in accordance with the guidelines of the IACUC at the Korea Brain Research Institute.

\section{Consent for publication}

Not applicable.

\section{Competing interests}

The authors declare that they have no competing interests.

\section{Author details}

'Dementia Research Group, Korea Brain Research Institute (KBRI), Daegu 41062, South Korea. ${ }^{2}$ Department of Brain \& Cognitive Sciences, DGIST, Daegu 42988, South Korea. ${ }^{3}$ Gwangju Center, Korea Basic Science Institute (KBSI), Gwangju 61886, South Korea. ${ }^{4}$ Research Division and Brain Research Core Facilities, Korea Brain Research Institute (KBRI), Daegu 41062, South Korea. ${ }^{5}$ School of Life Sciences, BK21 Plus KNU Creative BioResearch Group, Institute of Life Science \& Biotechnology, Kyungpook National University, Daegu 41566, South Korea. ${ }^{6}$ Brain Science and Engineering Institute, Kyungpook National University, Daegu 41944, South Korea. ${ }^{7}$ Department of Medical Biotechnology, Soonchunhyang University, Asan 31538, South Korea.

Received: 11 February 2020 Accepted: 23 September 2020

Published online: 14 October 2020

\section{References}

1. Scotter EL, Chen HJ, Shaw CE. TDP-43 Proteinopathy and ALS: insights into disease mechanisms and therapeutic targets. Neurotherapeutics. 2015;12: 352-63.

2. Buratti E. Functional significance of TDP-43 mutations in disease. Adv Genet. 2015;91:1-53. 
3. Neumann M, Sampathu DM, Kwong LK, Truax AC, Micsenyi MC, Chou TT, et al. Ubiquitinated TDP-43 in frontotemporal lobar degeneration and amyotrophic lateral sclerosis. Science. 2006;314:130-3.

4. Purice MD, Taylor JP. Linking hnRNP function to ALS and FTD pathology. Front Neurosci. 2018;12:326-37.

5. Kapeli K, Martinez FJ, Yeo GW. Genetic mutations in RNA-binding proteins and their roles in ALS. Hum Genet. 2017;136:1193-214.

6. Li Y, Ray P, Rao EJ, Shi C, Guo W, Chen X, et al. A drosophila model for TDP43 proteinopathy. Proc Natl Acad Sci U S A. 2010;107:3169-74.

7. Wils H, Kleinberger G, Janssens J, Pereson S, Joris G, Cuijt I, et al. TDP-43 transgenic mice develop spastic paralysis and neuronal inclusions characteristic of ALS and frontotemporal lobar degeneration. Proc Natl Acad Sci U S A. 2010;107:3858-63.

8. Xu YF, Gendron TF, Zhang YJ, Lin WL, D'Alton S, Sheng H, et al. Wild-type human TDP-43 expression causes TDP-43 phosphorylation, mitochondrial aggregation, motor deficits, and early mortality in transgenic mice. J Neurosci. 2010;30:10851-9.

9. Picher-Martel V, Valdmanis PN, Gould PV, Julien JP, Dupre N. From animal models to human disease: a genetic approach for personalized medicine in ALS. Acta Neuropathol Commun. 2016;4:70-98.

10. Lee $S$, Jeon YM, Cha SJ, Kim S, Kwon Y, Jo M, et al. PTK2/FAK regulates UPS impairment via SQSTM1/p62 phosphorylation in TARDBP/TDP-43 proteinopathies. Autophagy. 2019:1-17.

11. Kim HJ, Raphael AR, LaDow ES, McGurk L, Weber RA, Trojanowski JQ, et al. Therapeutic modulation of elF2alpha phosphorylation rescues TDP-43 toxicity in amyotrophic lateral sclerosis disease models. Nat Genet. 2014;46: $152-60$.

12. Shenouda M, Zhang AB, Weichert A, Robertson J. Mechanisms associated with TDP-43 neurotoxicity in ALS/FTLD. Adv Neurobiol. 2018;20:239-63.

13. Tremblay C, St-Amour I, Schneider J, Bennett DA, Calon F. Accumulation of transactive response DNA binding protein 43 in mild cognitive impairment and Alzheimer disease. J Neuropathol Exp Neurol. 2011;70:788-98.

14. Mackenzie IR, Neumann M, Baborie A, Sampathu DM, Du Plessis D, Jaros E, et al. A harmonized classification system for FTLD-TDP pathology. Acta Neuropathol. 2011;122:111-3.

15. Joyce PI, Fratta P, Fisher EM, Acevedo-Arozena A. SOD1 and TDP-43 animal models of amyotrophic lateral sclerosis: recent advances in understanding disease toward the development of clinical treatments. Mamm Genome. 2011;22:420-48.

16. Boillee S, Yamanaka K, Lobsiger CS, Copeland NG, Jenkins NA, Kassiotis G, et al. Onset and progression in inherited ALS determined by motor neurons and microglia. Science. 2006;312:1389-92.

17. Beers DR, Henkel JS, Xiao Q, Zhao W, Wang J, Yen AA, et al. Wild-type microglia extend survival in PU.1 knockout mice with familial amyotrophic lateral sclerosis. Proc Natl Acad Sci U S A. 2006;103:16021-6.

18. Yamanaka K, Chun SJ, Boillee S, Fujimori-Tonou N, Yamashita H, Gutmann $\mathrm{DH}$, et al. Astrocytes as determinants of disease progression in inherited amyotrophic lateral sclerosis. Nat Neurosci. 2008;11:251-3.

19. Di Giorgio FP, Carrasco MA, Siao MC, Maniatis T, Eggan K. Non-cell autonomous effect of glia on motor neurons in an embryonic stem cellbased ALS model. Nat Neurosci. 2007:10:608-14.

20. Ilieva H, Polymenidou M, Cleveland DW. Non-cell autonomous toxicity in neurodegenerative disorders: ALS and beyond. J Cell Biol. 2009;187:761-72.

21. Tong J, Huang C, Bi F, Wu Q, Huang B, Liu X, et al. Expression of ALS-linked TDP-43 mutant in astrocytes causes non-cell-autonomous motor neuron death in rats. EMBO J. 2013;32:1917-26.

22. Traves PG, Pardo V, Pimentel-Santillana M, Gonzalez-Rodriguez A, Mojena M, Rico D, et al. Pivotal role of protein tyrosine phosphatase 1B (PTP1B) in the macrophage response to pro-inflammatory and anti-inflammatory challenge. Cell Death Dis. 2014;5:e1125.

23. Lu X, Malumbres $R$, Shields B, Jiang X, Sarosiek KA, Natkunam Y, et al. PTP1B is a negative regulator of interleukin 4-induced STAT6 signaling. Blood. 2008;112:4098-108.

24. Tsunekawa T, Banno R, Mizoguchi A, Sugiyama M, Tominaga T, Onoue T, et al. Deficiency of PTP1B attenuates hypothalamic inflammation via activation of the JAK2-STAT3 pathway in microglia. EBioMedicine. 2017;16: 172-83

25. Song GJ, Jung $M$, Kim JH, Park $H$, Rahman MH, Zhang $S$, et al. A novel role for protein tyrosine phosphatase $1 \mathrm{~B}$ as a positive regulator of neuroinflammation. J Neuroinflammation. 2016:13:86-99.
26. Jeon YM, Lee S, Kim S, Kwon Y, Kim K, Chung CG, et al. Neuroprotective effects of protein tyrosine phosphatase $1 \mathrm{~b}$ inhibition against ER stressinduced toxicity. Mol Cells. 2017:40:280-90.

27. Enokido Y, Akaneya Y, Niinobe M, Mikoshiba K, Hatanaka H. Basic fibroblast growth factor rescues CNS neurons from cell death caused by high oxygen atmosphere in culture. Brain Res. 1992:599:261-71.

28. Araki W, Yuasa K, Takeda S, Shirotani K, Takahashi K, Tabira T. Overexpression of presenilin-2 enhances apoptotic death of cultured cortical neurons. Ann N Y Acad Sci. 2000;920:241-4

29. McCarthy KD, de Vellis J. Preparation of separate astroglial and oligodendroglial cell cultures from rat cerebral tissue. J Cell Biol. 1980;85: 890-902.

30. Livak KJ, Schmittgen TD. Analysis of relative gene expression data using real-time quantitative PCR and the 2(-Delta Delta C(T)) method. Methods. 2001;25:402-8.

31. Lantz RC, Lemus R, Lange RW, Karol MH. Rapid reduction of intracellular glutathione in human bronchial epithelial cells exposed to occupational levels of toluene diisocyanate. Toxicol Sci. 2001;60:348-55.

32. Bergenheim F, Seidelin JB, Pedersen MT, Mead BE, Jensen KB, Karp JM, et al Fluorescence-based tracing of transplanted intestinal epithelial cells using confocal laser endomicroscopy. Stem Cell Res Ther. 2019;10:148-59.

33. Cashman NR, Durham HD, Blusztajn JK, Oda K, Tabira T, Shaw IT, et al. Neuroblastoma x spinal cord (NSC) hybrid cell lines resemble developing motor neurons. Dev Dyn. 1992;194:209-21.

34. Maier O, Bohm J, Dahm M, Bruck S, Beyer C, Johann S. Differentiated NSC34 motoneuron-like cells as experimental model for cholinergic neurodegeneration. Neurochem Int. 2013;62:1029-38.

35. Johann S, Dahm M, Kipp M, Zahn U, Beyer C. Regulation of choline acetyltransferase expression by 17 beta-oestradiol in NSC-34 cells and in the spinal cord. J Neuroendocrinol. 2011;23:839-48.

36. Dranka BP, Benavides GA, Diers AR, Giordano S, Zelickson BR, Reily C, et al. Assessing bioenergetic function in response to oxidative stress by metabolic profiling. Free Radic Biol Med. 2011;51:1621-35.

37. Pandey UB, Nie Z, Batlevi Y, McCray BA, Ritson GP, Nedelsky NB, et al. HDAC6 rescues neurodegeneration and provides an essential link between autophagy and the UPS. Nature. 2007:447:859-63.

38. Sun W, Lee S, Huang X, Liu S, Inayathullah M, Kim KM, et al. Attenuation of synaptic toxicity and MARK4/PAR1-mediated tau phosphorylation by methylene blue for Alzheimer's disease treatment. Sci Rep. 2016;6:34784-93.

39. Feany MB, Bender WW. A drosophila model of Parkinson's disease. Nature. 2000;404:394-8

40. Ito $Y$, Banno $R$, Hagimoto S, Ozawa $Y$, Arima H, Oiso Y. TNFalpha increases hypothalamic PTP1B activity via the NFkappaB pathway in rat hypothalamic organotypic cultures. Regul Pept. 2012;174:58-64.

41. Zhao W, Beers DR, Bell S, Wang J, Wen S, Baloh RH, et al. TDP-43 activates microglia through NF-kappaB and NLRP3 inflammasome. Exp Neurol. 2015; 273:24-35.

42. Chen L, Deng H, Cui H, Fang J, Zuo Z, Deng J, et al. Inflammatory responses and inflammation-associated diseases in organs. Oncotarget. 2018;9:7204-18

43. Wang FW, Hao HB, Zhao SD, Zhang YM, Liu Q, Liu HJ, et al. Roles of activated astrocyte in neural stem cell proliferation and differentiation. Stem Cell Res. 2011;7:41-53.

44. Sun L, Li Y, Jia X, Wang Q, Li Y, Hu M, et al. Neuroprotection by IFN-gamma via astrocyte-secreted IL-6 in acute neuroinflammation. Oncotarget. 2017;8: 40065-78.

45. Kia A, McAvoy K, Krishnamurthy K, Trotti D, Pasinelli P. Astrocytes expressing ALS-linked mutant FUS induce motor neuron death through release of tumor necrosis factor-alpha. Glia. 2018:66:1016-33.

46. Shi P, Gal J, Kwinter DM, Liu X, Zhu H. Mitochondrial dysfunction in amyotrophic lateral sclerosis. Biochim Biophys Acta. 1802;2010:45-51.

47. Gautier CA, Corti O, Brice A. Mitochondrial dysfunctions in Parkinson's disease. Rev Neurol (Paris). 2014;170:339-43.

48. Birnbaum JH, Wanner D, Gietl AF, Saake A, Kundig TM, Hock C, et al. Oxidative stress and altered mitochondrial protein expression in the absence of amyloid-beta and tau pathology in iPSC-derived neurons from sporadic Alzheimer's disease patients. Stem Cell Res. 2018;27:121-30.

49. Wang W, Li L, Lin WL, Dickson DW, Petrucelli L, Zhang T, et al. The ALS disease-associated mutant TDP-43 impairs mitochondrial dynamics and function in motor neurons. Hum Mol Genet. 2013:22:4706-19. 
50. Cozzolino M, Carri MT. Mitochondrial dysfunction in ALS. Prog Neurobiol. 2012:97:54-66.

51. Kawamata H, Peixoto P, Konrad C, Palomo G, Bredvik K, Gerges M, et al. Mutant TDP-43 does not impair mitochondrial bioenergetics in vitro and in vivo. Mol Neurodegener. 2017;12:37-51.

52. Davis SA, Itaman S, Khalid-Janney CM, Sherard JA, Dowell JA, Cairns NJ, et al. TDP-43 interacts with mitochondrial proteins critical for mitophagy and mitochondrial dynamics. Neurosci Lett. 2018;678:8-15.

53. Steketee MB, Moysidis SN, Weinstein JE, Kreymerman A, Silva JP, lqbal S, et al. Mitochondrial dynamics regulate growth cone motility, guidance, and neurite growth rate in perinatal retinal ganglion cells in vitro. Invest Ophthalmol Vis Sci. 2012;53:7402-11.

54. Nguyen HTN, Kato H, Masuda K, Yamaza H, Hirofuji Y, Sato H, et al. Impaired neurite development associated with mitochondrial dysfunction in dopaminergic neurons differentiated from exfoliated deciduous toothderived pulp stem cells of children with autism spectrum disorder. Biochem Biophys Rep. 2018;16:24-31.

55. Bramham CR, Wells DG. Dendritic mRNA: transport, translation and function. Nat Rev Neurosci. 2007:8:776-89.

56. Benkler C, Ben-Zur T, Barhum Y, Offen D. Altered astrocytic response to activation in SOD1(G93A) mice and its implications on amyotrophic lateral sclerosis pathogenesis. Glia. 2013;61:312-26.

57. Eggett CJ, Crosier S, Manning P, Cookson MR, Menzies FM, McNeil CJ, et al. Development and characterisation of a glutamate-sensitive motor neurone cell line. J Neurochem. 2000;74:1895-902.

58. Panayidou S, Apidianakis Y. Regenerative inflammation: lessons from drosophila intestinal epithelium in health and disease. Pathogens. 2013;2:209-31.

59. Hoffmann JA. The immune response of drosophila. Nature. 2003;426:33-8.

60. Brennan CA, Anderson KV. Drosophila: the genetics of innate immune recognition and response. Annu Rev Immunol. 2004;22:457-83.

61. Minakhina S, Steward R. Nuclear factor-kappa B pathways in drosophila. Oncogene. 2006;25:6749-57.

62. Leal-Lasarte MM, Franco JM, Labrador-Garrido A, Pozo D, Roodveldt C. Extracellular TDP-43 aggregates target MAPK/MAK/MRK overlapping kinase (MOK) and trigger caspase-3/L-18 signaling in microglia. FASEB J. 2017;31: 2797-816.

63. Haidet-Phillips AM, Hester ME, Miranda CJ, Meyer K, Braun L, Frakes A, et al. Astrocytes from familial and sporadic ALS patients are toxic to motor neurons. Nat Biotechnol. 2011;29:824-8.

64. Qian K, Huang H, Peterson A, Hu B, Maragakis NJ, Ming GL, et al. Sporadic ALS astrocytes induce neuronal degeneration in vivo. Stem Cell Reports. 2017:8:843-55

65. Song GJ, Kim J, Kim JH, Song S, Park H, Zhang ZY, et al. Comparative analysis of protein tyrosine phosphatases regulating microglial activation. Exp Neurobiol. 2016;25:252-61.

66. Migheli A, Piva R, Atzori C, Troost D. Schiffer D: c-Jun, JNK/SAPK kinases and transcription factor NF-kappa B are selectively activated in astrocytes, but not motor neurons, in amyotrophic lateral sclerosis. J Neuropathol Exp Neurol. 1997;56:1314-22.

67. Swarup V, Phaneuf D, Dupre N, Petri S, Strong M, Kriz J, et al. Deregulation of TDP-43 in amyotrophic lateral sclerosis triggers nuclear factor kappaBmediated pathogenic pathways. J Exp Med. 2011;208:2429-47.

68. Yamasaki Y, Matsuura N, Shozuhara H, Onodera H, Itoyama Y, Kogure K. Interleukin-1 as a pathogenetic mediator of ischemic brain damage in rats. Stroke. 1995;26:676-81.

69. Lawrence $C B$, Allan SM, Rothwell NJ. Interleukin-1 beta and the interleukin-1 receptor antagonist act in the striatum to modify excitotoxic brain damage in the rat. Eur J Neurosci. 1998;10:1188-95.

70. Hu Y, Cao C, Qin XY, Yu Y, Yuan J, Zhao Y, et al. Increased peripheral blood inflammatory cytokine levels in amyotrophic lateral sclerosis: a meta-analysis study. Sci Rep. 2017;7:9094-102.

71. Chen X, Hu Y, Cao Z, Liu Q, Cheng Y. Cerebrospinal fluid inflammatory cytokine aberrations in Alzheimer's disease, Parkinson's disease and amyotrophic lateral sclerosis: a systematic review and meta-analysis. Front Immunol. 2018;9:2122-31.

72. Vandoorne T, De Bock K, Van Den Bosch L. Energy metabolism in ALS: an underappreciated opportunity? Acta Neuropathol. 2018;135:489-509.

73. Shan X, Chiang PM, Price DL, Wong PC. Altered distributions of Gemini of coiled bodies and mitochondria in motor neurons of TDP-43 transgenic mice. Proc Natl Acad Sci U S A. 2010;107:16325-30.
74. Khalil B, Cabirol-Pol MJ, Miguel L, Whitworth AJ, Lecourtois M, Lievens JC. Enhancing Mitofusin/Marf ameliorates neuromuscular dysfunction in drosophila models of TDP-43 proteinopathies. Neurobiol Aging. 2017;54:71-83.

75. Lu J, Duan W, Guo Y, Jiang H, Li Z, Huang J, et al. Mitochondrial dysfunction in human TDP-43 transfected NSC34 cell lines and the protective effect of dimethoxy curcumin. Brain Res Bull. 2012;89:185-90.

76. Madji Hounoum B, Vourc'h P, Felix R, Corcia P, Patin F, Gueguinou M, et al. NSC-34 motor neuron-like cells are unsuitable as experimental model for glutamate-mediated excitotoxicity. Front Cell Neurosci. 2016;10:118-29.

77. Kounatidis I, Chtarbanova S. Role of glial immunity in lifespan determination: a drosophila perspective. Front Immunol. 2018;9:1362-8.

78. Zhan L, Xie Q, Tibbetts RS. Opposing roles of $p 38$ and JNK in a drosophila model of TDP-43 proteinopathy reveal oxidative stress and innate immunity as pathogenic components of neurodegeneration. Hum Mol Genet. 2015; 24:757-72.

79. Joardar A, Menzl J, Podolsky TC, Manzo E, Estes PS, Ashford S, et al. PPAR gamma activation is neuroprotective in a drosophila model of ALS based on TDP-43. Hum Mol Genet. 2015;24:1741-54.

80. Estes PS, Daniel SG, McCallum AP, Boehringer AV, Sukhina AS, Zwick RA, et al. Motor neurons and glia exhibit specific individualized responses to TDP-43 expression in a drosophila model of amyotrophic lateral sclerosis. Dis Model Mech. 2013:6:721-33.

81. Krug L, Chatterjee N, Borges-Monroy R, Hearn S, Liao WW, Morrill K, et al. Retrotransposon activation contributes to neurodegeneration in a drosophila TDP-43 model of ALS. PLoS Genet. 2017;13:e1006635.

82. Freeman MR. Drosophila central nervous system glia. Cold Spring Harb Perspect Biol. 2015;7

83. Kerru N, Singh-Pillay A, Awolade P, Singh P. Current anti-diabetic agents and their molecular targets: a review. Eur J Med Chem. 2018;152:436-88.

\section{Publisher's Note}

Springer Nature remains neutral with regard to jurisdictional claims in published maps and institutional affiliations.

\section{Ready to submit your research? Choose BMC and benefit from:}

- fast, convenient online submission

- thorough peer review by experienced researchers in your field

- rapid publication on acceptance

- support for research data, including large and complex data types

- gold Open Access which fosters wider collaboration and increased citations

- maximum visibility for your research: over $100 \mathrm{M}$ website views per year

At BMC, research is always in progress.

Learn more biomedcentral.com/submissions 